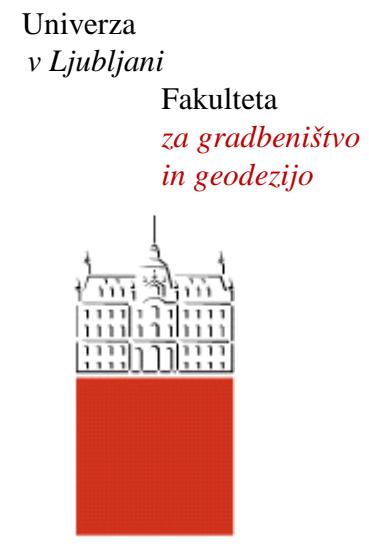

Jamova 2

1000 Ljubljana, Slovenija http://www3.fgg.uni-lj.si//

\section{DRUGG - Digitalni repozitorij UL FGG http://drugg.fgg.uni-lj.si/}

Ta članek je avtorjeva zadnja recenzirana različica, kot je bila sprejeta po opravljeni recenziji.

Prosimo, da se pri navajanju sklicujte na bibliografske podatke, kot je navedeno:
University
of Ljubljana

Faculty of

Civil and Geodetic

Engineering

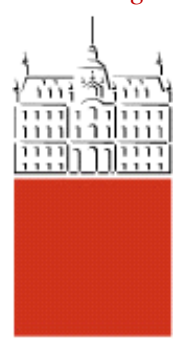

Jamova 2

SI - 1000 Ljubljana, Slovenia

http://www3.fgg.uni-lj.si/

DRUGG - The Digital Repository http://drugg.fgg.uni-lj.si/

This version of the article is author's manuscript as accepted for publishing after the review process.

When citing, please refer to the publisher's bibliographic information as follows:

Brank, B., Korelc, J., Ibrahimbegović, A. 2002.Nonlinear shell problem formulation accounting for through-the-thickness stretching and its finite element implementation. Computers and Structures 80, 9-10: 699-717.

http://www.journals.elsevier.com/computers-and-structures 


\title{
Nonlinear shell problem formulation accounting for through-the-thickness stretching and its finite element implementation
}

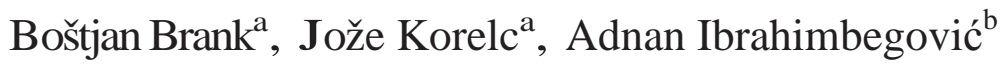 \\ a Faculty of Civil and Geodetic Engineering, University of Ljubljana, Jamova 2, 1000 Ljubljana, Slovenia \\ b Ecole Normale Superieure de Cachan, LMT, 61, av. du President Wilson, 94235 Cachan Cedex, France
}

\begin{abstract}
We discuss a theoretical formulation of shell model accounting for through-the-thickness stretching, which allows for large deformations and direct use of $3 \mathrm{~d}$ constitutive equations. Three different possibilities for implementing this model within the framework of the finite element method are examined: one leading to 7 nodal parameters and the remaining two to 6 nodal parameters. The 7-parameter shell model with no simplification of kinematic terms is compared to the 7-parameter shell model which exploits usual simplifications of the Green-Lagrange strains. Two different ways of implementing the incompatible mode method for reducing the number of parameters to 6 are presented. One implementation uses an additive decomposition of the strains and the other an additive decomposition of the deformation gradient. Several numerical examples are given to illustrate performance of the shell elements developed herein.
\end{abstract}

Keywords: Nonlinear shells; Thick shells; Thickness change; Incompatible modes

\section{Introduction}

The very recent research on the shell problem in the computational mechanics community is closing the gap between the classical shell model (which is basically $2 \mathrm{~d}$ ) and the classical $3 \mathrm{~d}$ solid model. While the later was mastered long ago, the former-the nonlinear analysis of shells-has mainly been restricted to the shell models which are naturally placed within the theoretical framework of the Cosserat surface (e.g. see [26]). Even in such a simplified setting the numerical implementation issues have been finally settled fairly recently (e.g. see [19] for a recent review). With both types of numerical models available it was natural to try to address an always present need to produce a model which would fit in between the shell (possibly retaining its computational efficiency) and the $3 \mathrm{~d}$ solid.

A model of that kind can serve well in representing a stress variation in through-the-thickness direction, and eventual boundary layer effects. More importantly, it can directly use the $3 \mathrm{~d}$ version of constitutive relations and thus eliminate eventual complexities stemming from imposing the zero-through-the-thickness-stress restriction on any constitutive model. It is desirable that such a shell model (further referred to as $3 \mathrm{~d}$ shell) be capable of recovering the $2 \mathrm{~d}$ shell behavior in the limit case of thin shell, as well as be considerably better in recovering true stress field for a thick shell than the usual thick shell model of Reissner-Mindlin type. 
Few different approaches have been explored recently. The first group of works (e.g. [1-3,6,29]) started with the Reissner-Mindlin shell model (with three displacements of the mid-surface and two rotation parameters of the shell director typically used for smooth shells) enriching it further by a desired number of parameters to permit a reliable representation of throughthe-thickness stretching. Those parameters are either independent kinematic variables, or strain variables constructed in the framework of the enhanced assumed strain (EAS) method, which are further eliminated at the finite element level. Second group (e.g. [7,13,28]) went along a similar path, but instead of describing shell director deformation with two rotation parameters they rather used three components of so-called difference vector. So-developed shell models posses no rotation degrees of freedom. Final group to be mentioned (e.g. see $[15,21,27])$ preferred to take a solid element as the basis for their developments. They reduced the shell-like features of the so-developed elements to special treatment of shear deformation along with the modifications for through-the-thickness stretching.

For any of the $3 \mathrm{~d}$ shell models mentioned above the use of the fully $3 \mathrm{~d}$ constitutive equations should preferably be accompanied by a linear variation of the through-the-thickness deformation component. This imposes two additional kinematic parameters for models with rotations and one for models with displacements only. One arrives at a 7-parameter shell theory. If one intends to decrease the computational efficiency and, more importantly, simplify the issues of the corresponding boundary conditions, the method of incompatible modes (e.g. see [16,17,31]) ought to be employed in order to reduce the number of parameters to 6 .

We focus in this work on two questions. First, we study the difference between a 7-parameter theory where the exact expressions are used for the Green-Lagrange strain measures versus the shell theory where the usual simplifications (e.g. see $[10,12]$ ) are carried out by neglecting certain terms. The former of these two models can be developed without difficulty mostly for our use of symbolic manipulation (see $[22,24])$. The second study is oriented towards two possible implementations of the method of incompatible modes: one with an additive decomposition of strains (e.g. [16]) versus the other with an additive decomposition of the deformation gradient which leads to a multiplicative decomposition of strains (e.g. [17])

The outline of this paper is as follows. In Section 2 we lay the governing equations of the 7-parameter shell model. Two different variants of the incompatible mode methods are presented in Section 3. In Section 4 we provide some details of the numerical implementation. Several numerical examples are presented in Section 5 and the concluding remarks are given in Section 6.

\section{Shell theory with 7 parameters}

In this section we first elaborate upon a shell formulation which employs the Reissner-Mindlin hypothesis that a straight fiber remains straight, but with enhanced, higher-order variation of the through-the-thickness displacement components. We then move on to develop the corresponding form of the Green-Lagrange strain measures. To complete the theory we deal with the simplest set of hyperelastic constitutive equations: the St. Venant-Kirchhoff (SVK) and the neo-Hookean (NH) materials. Finally the equilibrium equations are presented in their weak form along with their consistent linearization.

Contrary to the classical Reissner-Mindlin kinematics (incapable of accounting for through-the-thickness deformation), we set to develop an enriched kinematic field in order to extend the potential application domain of the developed shell model. To that end, the shell position vector from the initial configuration

$x\left(\xi^{1}, \xi^{2}, \zeta\right)=\varphi_{0}\left(\xi^{1}, \xi^{2}\right)+\zeta \frac{h_{0}}{2} \mathrm{~g}\left(\xi^{1}, \xi^{2}\right)$

with

$\|\mathrm{g}\|=1, \quad\left(\xi^{1}, \xi^{2}\right) \subset \mathscr{A} \subset \mathbb{R}^{2}, \quad \zeta \in[-1,1]$

(where $\xi^{1}, \xi^{2}$ and $\zeta$ are natural or convected coordinates, $\boldsymbol{\varphi}_{0}$ is the position vector of the shell middle surface, $h_{0}$ is the initial constant shell thickness, $\mathscr{A}$ is the domain of the shell middle surface parametrization, and $g$ is the initial unit normal or shell director) is transformed into its counterpart at the deformed configuration as

$$
\begin{aligned}
x\left(\xi^{1}, \xi^{2}, \zeta\right)= & \varphi\left(\xi^{1}, \xi^{2}\right)+\zeta \frac{h\left(\xi^{1}, \xi^{2}\right)}{2} \mathrm{a}\left(\xi^{1}, \xi^{2}\right) \\
& +\left[\zeta \frac{h\left(\xi^{1}, \xi^{2}\right)}{2}\right]^{2} \widetilde{q}\left(\xi^{1}, \xi^{2}\right) \mathrm{a}\left(\xi^{1}, \xi^{2}\right),
\end{aligned}
$$

with

$\|\mathrm{a}\|=1$

and

$\boldsymbol{\varphi}\left(\xi^{1}, \xi^{2}\right)=\boldsymbol{\varphi}_{0}\left(\xi^{1}, \xi^{2}\right)+\mathrm{u}\left(\xi^{1}, \xi^{2}\right)$.

In (3) and (5) $\mathrm{u}$ is the displacement vector providing a new position of the middle surface, $h$ is the current shell thickness, a is the current position of the shell director and $\widetilde{q}$ is the hierarchical term introducing the displacement quadratic variation in the through-the-thickness direction. Considering that we allow for thickness change in the direction of $\zeta$ coordinate $^{2}$ with

$\lambda\left(\xi^{1}, \xi^{2}\right)=\frac{h\left(\xi^{1}, \xi^{2}\right)}{h_{0}}$,

\footnotetext{
${ }^{2}$ Note that $\zeta$ coordinate is not perpendicular to the middle surface at the deformed configuration.
} 
we may write (3) as

$$
\begin{gathered}
x\left(\xi^{1}, \xi^{2}, \zeta\right)=\boldsymbol{\varphi}\left(\xi^{1}, \xi^{2}\right)+\zeta \frac{h_{0}}{2} \lambda\left(\xi^{1}, \xi^{2}\right) \mathrm{a}\left(\xi^{1}, \xi^{2}\right) \\
+\zeta^{2} \frac{h_{0}^{2}}{4} q\left(\xi^{1}, \xi^{2}\right) \mathrm{a}\left(\xi^{1}, \xi^{2}\right),
\end{gathered}
$$

where

$q=\lambda \widetilde{q}$.

We note that the structure of the term for quadratic variation of displacements in through-the-thickness direction chosen in (7) is just one of the several possibilities. To simplify the notation we further rewrite (7) as ${ }^{3}$

$\mathrm{x}\left(\xi^{1}, \xi^{2}, \zeta\right)=\boldsymbol{\varphi}\left(\xi^{1}, \xi^{2}\right)+\zeta \mathrm{d}\left(\xi^{1}, \xi^{2}\right)+\zeta^{2} \mathrm{f}\left(\xi^{1}, \xi^{2}\right)$,

where

$\mathrm{d}=\frac{h_{0}}{2} \lambda \mathrm{a}, \quad \mathrm{f}=\frac{h_{0}^{2}}{4} q \mathrm{a}$.

The position of the shell director a is defined by two rotational parameters, which are in this work two components of the total material rotation vector $\vartheta$

$\mathrm{a}=\mathrm{a}\left(\vartheta^{1}, \vartheta^{2}\right)$

(e.g. see [10] or [11] for details).

The configuration space consistent with the choice of kinematics indicated in (3) has 7 parameters

$C=\left\{\begin{array}{l}\Phi=(\boldsymbol{\varphi}, \mathrm{a}, \lambda, q)\left|\mathscr{A} \rightarrow \mathbb{R}^{3} \times S^{2} \times \mathbb{R}^{+} \times \mathbb{R}\right| \\ \boldsymbol{\varphi}_{\mid \mathrm{\partial} \boldsymbol{L} \mathscr{A}}=\overline{\boldsymbol{\varphi}}, \mathrm{a}_{\mid \mathrm{\partial} \mathrm{a} \mathscr{A}}=\overline{\mathrm{a}}, \lambda_{\mid \partial \lambda \mathscr{A}}=\bar{\lambda}, q_{\mid \partial q \mathscr{A}}=\bar{q}\end{array}\right\}$,

where $\partial \varphi \mathscr{A}, \ldots, \partial q \mathscr{A}$ are parts of the shell boundary where the corresponding variable value is prescribed. In (12), it is indicated that the unit vector a belongs to a unit sphere manifold, which imposes a special treatment of finite rotations (e.g. see [30] or [20]).

Departing from the classical exposition on the subject (e.g. [26]), which reduces the shell theory to a $2 \mathrm{~d}$ setting, we keep herein the fully $3 \mathrm{~d}$ picture. Consequently, the choice of the coordinates in the shell-deformed configuration leads to the following vector basis:

$$
\begin{aligned}
& a_{\alpha}=\frac{\partial \mathrm{x}}{\partial \xi^{\alpha}}=\boldsymbol{\varphi}_{, \alpha}+\zeta \mathrm{d}_{, \alpha}+\zeta^{2} \mathrm{f}_{, \alpha}, \\
& \mathrm{a}_{3}=\frac{\partial \mathrm{x}}{\partial \zeta}=\mathrm{d}+2 \zeta \mathrm{f},
\end{aligned}
$$

where $(\cdot)_{, \alpha}=\left(\partial(\cdot) / \partial \xi^{\alpha}\right), \alpha=1,2$ and

$\mathrm{d}_{, \alpha}=\frac{h_{0}}{2}\left(\lambda_{, \alpha} \mathrm{a}+\lambda \mathrm{a}_{, \alpha}\right), \quad \mathrm{f}_{, \alpha}=\frac{h_{0}^{2}}{4}\left(q, \alpha \mathrm{a}+q \mathrm{a}_{, \alpha}\right)$.

\footnotetext{
${ }^{3}$ Eq. (9) can be regarded as a two-term approximation of $\mathrm{x}=\boldsymbol{\varphi}+\sum_{n=1}^{\infty} \zeta^{n} \mathrm{~d}_{n}$ given by Naghdi [26, p. 466] to derive a shell theory from the $3 \mathrm{~d}$ solid; see also [25].
}

The Green-Lagrange strains may be written as

$\mathrm{E}=\frac{1}{2}\left(\mathrm{~F}^{\mathrm{T}} \mathrm{F}-\mathbf{1}\right)=\frac{1}{2}(\mathrm{C}-\mathbf{1})=E_{i j} \mathrm{~g}^{i} \otimes \mathrm{g}^{j}$,

where $\mathrm{F}$ is the deformation gradient, $\mathbf{1}$ is the unit tensor, $\mathrm{C}$ is the right Cauchy-Green stretch tensor and $\mathrm{g}^{i}$ are contravariant base vectors of the initial configuration, defined as $\mathrm{g}_{i} \cdot \mathrm{g}^{j}=\delta_{i}^{j}$, where $\delta_{i}^{j}$ is Kronecker delta symbol. Base vectors $g_{i}$ follow from (1) as

$$
\begin{aligned}
& \mathrm{g}_{\alpha}=\frac{\partial \mathrm{X}}{\partial \xi^{\alpha}}=\boldsymbol{\varphi}_{0, \alpha}+\zeta \frac{h_{0}}{2} \mathrm{~g}_{, \alpha}, \\
& \mathrm{g}_{3}=\frac{\partial \mathrm{X}}{\partial \zeta}=\frac{h_{0}}{2} \mathrm{~g} .
\end{aligned}
$$

Note that $\mathrm{g}^{3}=\left(2 / h_{0}\right) \mathrm{g}$. Strains in that basis are defined as $\left(\mathrm{F}=\mathrm{a}_{i} \otimes \mathrm{g}^{i}\right.$ and $\left.\mathbf{1}=\mathrm{g}_{i} \cdot \mathrm{g}_{j} \mathrm{~g}^{i} \otimes \mathrm{g}^{j}\right)$

$$
\begin{aligned}
& E_{i j}=\frac{1}{2}\left(\mathrm{a}_{i} \cdot \mathrm{a}_{j}-\mathrm{g}_{i} \cdot \mathrm{g}_{j}\right) \\
& =H_{i j}+\zeta K_{i j}+\zeta^{2} L_{i j}+\zeta^{3} M_{i j}+\zeta^{4} N_{i j},
\end{aligned}
$$

with their explicit forms ${ }^{4}$ obtained by using (13) and (14)

$$
\begin{aligned}
& H_{\alpha \beta}=\frac{1}{2}\left(\boldsymbol{\varphi}_{, \alpha} \cdot \boldsymbol{\varphi}_{, \beta}-\boldsymbol{\varphi}_{0, \alpha} \cdot \boldsymbol{\varphi}_{0, \beta}\right), \\
& H_{\alpha 3}=\frac{1}{2}(\boldsymbol{\varphi}_{, \alpha} \cdot \mathrm{d}-\frac{h_{0}}{2} \underbrace{\boldsymbol{\varphi}_{0, \alpha} \cdot \mathrm{g}}_{0}) \\
& H_{33}=\frac{1}{2}\left(\mathrm{~d} \cdot \mathrm{d}-\frac{h_{0}^{2}}{4}\right), \\
& K_{\alpha \beta}=\frac{1}{2}\left(\boldsymbol{\varphi}_{, \alpha} \cdot \mathrm{d}_{, \beta}+\boldsymbol{\varphi}_{, \beta} \cdot \mathrm{d}_{, \alpha}-\frac{h_{0}}{2} \boldsymbol{\varphi}_{0, \alpha} \cdot \mathrm{g}_{, \beta}-\frac{h_{0}}{2} \boldsymbol{\varphi}_{0, \beta} \cdot \mathrm{g}_{, \alpha}\right), \\
& K_{\alpha 3}=\frac{1}{2}(\mathrm{~d}{ }_{, \alpha} \cdot \mathrm{d}+2 \boldsymbol{\varphi}_{, \alpha} \cdot \mathrm{f}-\frac{h_{0}^{2}}{4} \underbrace{\mathrm{g}_{, \alpha} \cdot \mathrm{g}}_{0}) \\
& K_{33}=\frac{1}{2}(4 \mathrm{f} \cdot \mathrm{d}),
\end{aligned}
$$

$L_{\alpha \beta}=\frac{1}{2}\left(\mathrm{~d}_{, \alpha} \cdot \mathrm{d}_{, \beta}+\boldsymbol{\varphi}_{, \alpha} \cdot \mathrm{f}_{, \beta}+\boldsymbol{\varphi}_{, \beta} \cdot \mathrm{f}_{, \alpha}-\frac{h_{0}^{2}}{4} \mathrm{~g}_{, \alpha} \cdot \mathrm{g}_{, \beta}\right)$,

$L_{\alpha 3}=\frac{1}{2}\left(2 \mathrm{~d}_{, \alpha} \cdot \mathrm{f}+\mathrm{f}_{, \alpha} \cdot \mathrm{d}\right)$,

$L_{33}=\frac{1}{2}(4 \mathrm{f} \cdot \mathrm{f})$,

$M_{\alpha \beta}=\frac{1}{2}\left(\mathrm{~d}_{, \alpha} \cdot \mathrm{f}_{, \beta}+\mathrm{d}_{, \beta} \cdot \mathrm{f}_{, \alpha}\right)$,
$M_{\alpha 3}=\frac{1}{2}\left(2 \mathrm{f}_{, \alpha} \cdot \mathrm{f}\right)$
$M_{33}=0$

\footnotetext{
${ }^{4}$ The through-the-thickness coordinate in shell theories is usually defined as $\xi=\zeta\left(h_{0} / 2\right)$ having $(\cdot)_{3}=\partial(\cdot) / \partial \xi$. Since we work here with $\zeta$ coordinate and $(\cdot)_{3}=\partial(\cdot) / \partial \zeta$, we obtain for strains an additional term of $h_{0} / 2$ for each subscript 3 .
} 
$N_{\alpha \beta}=\frac{1}{2}\left(\mathrm{f}_{, \alpha} \cdot \mathrm{f}_{, \beta}\right)$,

$N_{\alpha 3}=0$,

$N_{33}=0$.

From the above expressions it can be seen that the inplane shell strains are of fourth order with respect to $\zeta$ coordinate, while the transverse shear strains and the transverse normal strain vary cubicly and quadratically, respectively.

Usual simplification carried out in the shell theory developments (see e.g. $[2,10,12,28]$ ) is to truncate expression (17) after the linear term, so that

$E_{i j} \rightarrow H_{i j}+\zeta K_{i j}$.

In this work we will develop a model with exact expressions for strains and a simplified model with constant and linear variation of strains through the thickness.

Having defined the kinematics for the chosen 7parameter shell model, we proceed with the constitutive equations. We will restrict ourself to a simplest set of hyperelastic materials: the SVK and the NH. The stored energy density function per unit initial volume of the SVK material is defined as

$W(\mathrm{E})=\frac{\lambda}{2}(\operatorname{tr} \mathrm{E})^{2}+\mu \operatorname{tr} \mathrm{E}^{2}$,

where

$\lambda=\frac{E v}{(1+v)(1-2 v)} \quad$ and $\quad \mu=\frac{E}{2(1+v)}$

are Lamé coefficients, $\operatorname{tr}(\cdot)$ is trace of tensor $(\cdot)$, and $E$ is the Green-Lagrange strain tensor deduced above. The stored energy density function for the NH material reads as

$W(\mathrm{E})=\frac{\lambda}{2}(J-1)^{2}+\mu\left(\frac{\operatorname{tr} C-3}{2}-\ln J\right)$,

where $J=\sqrt{\operatorname{det}[C]}$. No shear correction factors are used in the constitutive models. Derivation of (24) and (25) with respect to the strain tensor leads to expressions for the second Piola-Kirchhoff stress tensor. We have

$\mathrm{S}=\frac{\partial W}{\partial \mathrm{E}}=\lambda \operatorname{tr} \mathrm{E} \mathbf{1}+2 \mu \mathrm{E}=S^{i j} \mathrm{~g}_{i} \otimes \mathrm{g}_{j}$

for the SVK material and

$$
\begin{aligned}
\mathrm{S} & =2 \frac{\partial W}{\partial \mathrm{C}}=\lambda(J-1) J \mathrm{C}^{-1}+\mu\left(\mathbf{1}-\mathrm{C}^{-1}\right) \\
& =S^{i j} \mathrm{~g}_{i} \otimes \mathrm{g}_{j}
\end{aligned}
$$

for the NH material. Derivation of stresses with respect to strains gives the components of the constitutive tensor

$\mathrm{C}=\frac{\partial \mathrm{S}}{\partial \mathrm{E}}=\frac{\partial^{2} W(\mathrm{E})}{\partial \mathrm{E}^{2}}=\mathrm{C}^{i j k l} \mathrm{~g}^{i} \otimes \mathrm{g}^{j} \otimes \mathrm{g}^{k} \otimes \mathrm{g}^{l}$.
We can thus write the total potential energy for the present shell model in the same way as for the $3 \mathrm{~d}$ solid

$\Pi(\boldsymbol{\varphi}, \mathrm{a}, \lambda, q)=\int_{A} \int_{h_{0}} W[\mathrm{E}(\boldsymbol{\varphi}, \mathrm{a}, \lambda, q)] \mathrm{d} V+\Pi_{\mathrm{ext}}(\boldsymbol{\varphi})$,

where $A$ defines the shell middle surface and $\Pi_{\text {ext }}$ is the potential of the conservative external forces acting on the middle surface ${ }^{5}$ of the shell, which may be written as

$\Pi_{\text {ext }}(\varphi)=-\int_{A} h_{0} \rho_{0} \overline{\mathrm{b}} \cdot \boldsymbol{\varphi} \mathrm{d} A-\int_{A} \mathrm{p} \cdot \boldsymbol{\varphi} \mathrm{d} A-\int_{\partial A} \overline{\mathrm{t}} \cdot \boldsymbol{\varphi} \mathrm{d} s$.

In (30) $\bar{b}, p$ and $\overline{\mathrm{t}}$ are applied body forces, pressure forces and forces acting on the edges of the shell middlesurface, respectively, and $\rho_{0}$ is the initial $3 \mathrm{~d}$ mass density. Variation of (29) with respect to the independent kinematic variables leads to the weak form of equilibrium equations

$\int_{A} \int_{h_{0}} \frac{\partial W(\mathrm{E})}{\partial \mathrm{E}} \delta \mathrm{E} \mathrm{d} V=D \Pi_{\text {ext }}(\boldsymbol{\varphi}) \cdot \delta \boldsymbol{\varphi}$

where the variation of strains, $\delta \mathrm{E}=D \mathrm{E}(\Phi) \cdot \delta \Phi$, can be obtained by varying (18)-(22). Linearization of (31) gives the tangent operator

$\int_{A} \int_{h_{0}}[(\mathrm{C} \Delta \mathrm{E}) \delta \mathrm{E}+\mathrm{S}(\Delta \delta \mathrm{E})] \mathrm{d} V$

where $\Delta \mathrm{E}$ is the linearization of strains $\Delta \mathrm{E}=$ $D \mathrm{E}(\Phi) \cdot \Delta \Phi$, and $\Delta \delta \mathrm{E}$ is the linearization of the variation of strains $\Delta \delta \mathrm{E}=D[\delta \mathrm{E}(\Phi)] \cdot \Delta \Phi$.

\section{Shell theory with six parameters and method of incompatible modes}

The 7-parameter shell theory developed in the previous section is very much geared towards the applications of a shell-like structures, and it might be difficult to use it as a part of the model of a complex system. Therefore, we develop in this section an alternative implementation of the shell theory with through-thethickness stretching where the number of parameters is reduced to 6 , which might be easier to combine with solids. In order to accommodate the linear variation of the through-the-thickness stretch we resort to the method of incompatible modes. We obtain a shell finite

\footnotetext{
${ }^{5}$ The potential of the conservative external forces can be extended to the forces acting on the shell top and bottom surfaces, i.e. $\Pi_{\mathrm{ext}}=\Pi_{\mathrm{ext}}\left(\boldsymbol{\varphi}+\zeta \mathrm{d}+\zeta^{2} \mathrm{f}\right)$, where $\zeta= \pm 1$.
} 
element with six nodal parameters, which possesses an additional advantage of fitting easier into the standard finite element software architecture.

Two possible implementations of the incompatible mode method are considered: one with an additive decomposition of strains and the other with an additive decomposition of the deformation gradient, which leads to a multiplicative decomposition of strains. The former is simpler, but only acceptable for small strains, whereas the latter, although more complex to handle, is also applicable for large strains.

\subsection{Incompatible modes based on an additive decompo- sition of strains}

If one wants to recover a 6 -parameter ${ }^{6}$ shell theory, the through-the-thickness displacement variation ought not be more than linear. This results in the following deformed configuration position vector:

$$
\begin{aligned}
x\left(\xi^{1}, \xi^{2}, \zeta\right) & =\boldsymbol{\varphi}\left(\xi^{1}, \xi^{2}\right)+\zeta \frac{h_{0}}{2} \lambda\left(\xi^{1}, \xi^{2}\right) \mathrm{a}\left(\xi^{1}, \xi^{2}\right) \\
& =\boldsymbol{\varphi}\left(\xi^{1}, \xi^{2}\right)+\zeta \mathrm{d}\left(\xi^{1}, \xi^{2}\right),
\end{aligned}
$$

with $d$ already defined in (10). The corresponding base vectors are then

$$
\begin{aligned}
& \mathrm{a}_{\alpha}=\frac{\partial \mathbf{x}}{\partial \xi^{\alpha}}=\boldsymbol{\varphi}_{, \alpha}+\zeta \mathrm{d}_{, \alpha}, \\
& \mathrm{a}_{3}=\frac{\partial \mathrm{x}}{\partial \zeta}=\mathrm{d} .
\end{aligned}
$$

The initial configuration position vector and its derivatives remain the same as indicated in (1) and (16), respectively.

The configuration space of the shell model consistent with the choice of kinematics indicated in (33) has six parameters: three displacements of the middle surface, two rotation parameters defining the position of the shell director a and one through-the-thickness stretching parameter $\lambda$. It can be written as

$C=\left\{\begin{array}{l}\Phi=(\boldsymbol{\varphi}, \mathrm{a}, \lambda)\left|\mathscr{A} \rightarrow \mathbb{R}^{3} \times S^{2} \times \mathbb{R}^{+}\right|, \\ \boldsymbol{\varphi}_{\mid \hat{\mid} \boldsymbol{\varphi} \mathscr{A}}=\overline{\boldsymbol{\varphi}}, \mathrm{a}_{\mid \mathrm{a} \mathrm{a} \mathscr{A}}=\overline{\mathrm{a}}, \lambda_{\mid \partial \lambda \mathscr{A}}=\bar{\lambda} .\end{array}\right\}$

The Green-Lagrange strains for the 6-parameter model in the $\mathrm{g}^{i}$ base are then

$$
E_{i j}=\frac{1}{2}\left(\mathrm{a}_{i} \cdot \mathrm{a}_{j}-\mathrm{g}_{i} \cdot \mathrm{g}_{j}\right)=H_{i j}+\zeta K_{i j}+\zeta^{2} L_{i j},
$$

\footnotetext{
${ }^{6}$ The same notation is used in Sections 2 and 3, although some quantities of the 6-parameter shell theory (like $\mathbf{x}, \mathbf{a}_{i}$, some strains, etc.) are of different form than those of the 7-parameter shell theory.
}

where

$$
\begin{aligned}
& H_{\alpha \beta}=\frac{1}{2}\left(\boldsymbol{\varphi}_{, \alpha} \cdot \boldsymbol{\varphi}_{, \beta}-\boldsymbol{\varphi}_{0, \alpha} \cdot \boldsymbol{\varphi}_{0, \beta}\right), \\
& H_{\alpha 3}=\frac{1}{2}\left(\boldsymbol{\varphi}_{, \alpha} \cdot \mathrm{d}\right), \\
& H_{33}=\frac{1}{2}\left(\mathrm{~d} \cdot \mathrm{d}-\frac{h_{0}^{2}}{4}\right), \\
& K_{\alpha \beta}=\frac{1}{2}\left(\boldsymbol{\varphi}_{, \alpha} \cdot \mathrm{d}_{, \beta}+\boldsymbol{\varphi}_{, \beta} \cdot \mathrm{d}_{, \alpha}-\frac{h_{0}}{2} \boldsymbol{\varphi}_{0, \alpha} \cdot \mathrm{g}_{, \beta}-\frac{h_{0}}{2} \boldsymbol{\varphi}_{0, \beta} \cdot \mathrm{g}_{, \alpha}\right), \\
& K_{\alpha 3}=\frac{1}{2}\left(\mathrm{~d}_{, \alpha} \cdot \mathrm{d}\right), \\
& K_{33}=0,
\end{aligned}
$$

$$
\begin{aligned}
L_{\alpha \beta} & =\frac{1}{2}\left(\mathrm{~d}_{, \alpha} \cdot \mathrm{d}_{, \beta}-\frac{h_{0}^{2}}{4} \mathrm{~g}_{, \alpha} \cdot \mathrm{g}_{, \beta}\right), \\
L_{\alpha 3} & =0 \\
L_{33} & =0 .
\end{aligned}
$$

Through-the-thickness variation of the in-plane strains, the transverse shear strains and the transverse normal strain is quadratic, linear and constant, respectively. A problem arises from the zero value of $K_{33}$ in (38), which implies a constant value of $E_{33}$ strain. Namely, even for the simplest stress state of pure bending (equivalent to the patch test condition, e.g. see [33]) with the linear variation of in-plane strain components in through-thethickness direction, the plane stress state can never be reproduced for any non-zero value of Poisson's ratio, since

$$
S^{33}=\mathrm{C}^{33 \alpha \beta} \underbrace{E_{\alpha \beta}}_{\text {linear in } \zeta}+\mathrm{C}^{3333} \underbrace{E_{33}}_{\text {constant in } \zeta} \text {. }
$$

This kind of problem is often referred as Poisson's ratio stiffening (see e.g. $[8,13]$ ).

If one would like to employ a $3 \mathrm{~d}$ constitutive model for shells and still avoid Poisson's ratio stiffening, it is indispensable to use a linear variation of the $E_{33}$ strain component, which can be introduced by the incompatible mode method

$$
E_{i j} \rightarrow E_{i j}+\widetilde{E}_{i j}, \quad \widetilde{E}_{i j}= \begin{cases}0, & i, j=1 \text { or } 2, \\ 0, & i \text { or } j=3, \\ \zeta A_{33}, & i, j=3 .\end{cases}
$$

This modification can then be introduced into the energy functional governing the shell problem according to

$$
\begin{aligned}
\Pi(\underbrace{\boldsymbol{\varphi}, \mathrm{a}, \lambda}_{\Phi}, \widetilde{E}_{i j}, S^{i j}) \\
=\int_{A} \int_{h_{0}}\left\{\frac{1}{2}\left(E_{i j}+\widetilde{E}_{i j}\right) C^{i j k l}\left(E_{k l}+\widetilde{E}_{k l}\right)-S^{i j} \widetilde{E}_{i j}\right\} \mathrm{d} V \\
\quad-\Pi_{\mathrm{ext}}(\boldsymbol{\varphi}) .
\end{aligned}
$$


The second term in the integral in (42) represents the Lagrange multiplier modification forcing the enhancement $\widetilde{E}_{33}$ to disappear in the strong form of the problem. The same does not happen in the weak form, which can be written as

$$
\begin{aligned}
D \Pi\left(\Phi, \widetilde{E}_{i j}, S^{i j}\right) \cdot \delta \Phi \\
=\int_{A} \int_{h_{0}} \delta E_{i j} C^{i j k l}\left(E_{k l}+\widetilde{E}_{k l}\right) \mathrm{d} V-D \Pi_{\text {ext }}(\varphi) \cdot \delta \varphi=0,
\end{aligned}
$$

$$
\begin{aligned}
D \Pi & \left(\Phi, \widetilde{E}_{i j}, S^{i j}\right) \cdot \delta \widetilde{E}_{i j} \\
= & \int_{A} \int_{h_{0}} \delta \widetilde{E}_{i j}\left[-S^{i j}+C^{i j k l}\left(E_{k l}+\widetilde{E}_{k l}\right)\right] \mathrm{d} V=0,
\end{aligned}
$$

$D \Pi\left(\Phi, \widetilde{E}_{i j}, S^{i j}\right) \cdot \delta S^{i j}=\int_{A} \int_{h_{0}} \delta S^{i j} \widetilde{E}_{i j} \mathrm{~d} V=0$.

Expressions (43)-(45) can be simplified by assuming orthogonality of the chosen strain enhancement and the stress field, making the first term in each of the last two equations to disappear. Eq. (45) implies that one should have

$0=\int_{A} \int_{h_{0}} S^{33} \widetilde{E}_{33} \mathrm{~d} V=\int_{A} \int_{h_{0}} S^{33}\left(\zeta A_{33}\right) \mathrm{d} V$

if $S^{33}=\operatorname{funct}\left(\xi^{1}, \xi^{2}\right)$.

One has to ensure, however, that the constant through-the-thickness stress field is contained in the chosen stress variation thus ensuring the patch test condition (e.g. [32]) in the following form:

$\int_{\square} \int_{-1}^{1} \widetilde{E}_{33} \underbrace{j \mathrm{~d} \zeta \mathrm{d} \xi^{1} \mathrm{~d} \xi^{2}}_{\mathrm{d} V}=0$,

where $\square$ is a bi-unit square and $j$ is Jacobian of the transformation from the initial shell finite element configuration to a bi-unit cube $\left(j=\left(\operatorname{det}\left[\mathrm{g}_{i} \cdot \mathrm{g}_{j}\right]\right)^{1 / 2}\right)$. Interpolation of $A_{33}$ over the finite element may be chosen as

$$
\begin{aligned}
A_{33}\left(\xi^{1}, \xi^{2}\right) & =\frac{j_{0}}{j}\left(\alpha_{1}+\alpha_{2} \xi^{1}+\alpha_{3} \xi^{2}+\alpha_{4} \xi^{1} \xi^{2}\right) \\
& =\frac{j_{0}}{j} \mathrm{n}^{\mathrm{T}} \boldsymbol{\alpha}
\end{aligned}
$$

or otherwise with the bi-linear functions as

$$
\begin{aligned}
A_{33}\left(\xi^{1}, \xi^{2}\right)= & \frac{j_{0}}{j}\left[\widehat{\alpha}_{1} N_{1}\left(\xi^{1}, \xi^{2}\right)+\widehat{\alpha}_{2} N_{2}\left(\xi^{1}, \xi^{2}\right)\right. \\
& \left.+\widehat{\alpha}_{3} N_{3}\left(\xi^{1}, \xi^{2}\right)+\widehat{\alpha}_{4} N_{4}\left(\xi^{1}, \xi^{2}\right)\right] \\
= & \frac{j_{0}}{j} \widehat{n}^{\mathrm{T}} \widehat{\boldsymbol{\alpha}} .
\end{aligned}
$$

In (48) and (49) $j_{0}$ is Jacobian at the center of the finite element (at $\xi^{1}=\xi^{2}=\zeta=0$ ), vector

$$
\boldsymbol{\alpha}=\left\{\alpha_{1}, \alpha_{2}, \alpha_{3}, \alpha_{4}\right\}^{\mathrm{T}}
$$

is vector of four local element strain parameters ${ }^{7}$ associated with interpolation of $\widetilde{E}_{33}$, and $\mathrm{n}$ is vector of interpolation functions for $\alpha$. $N_{a}$ (with $a=1,2,3,4$ ) are standard bi-linear interpolation functions for 4noded finite element (which is also a particular choice for the implementation of the present shell theory; see Section 4)

$$
\begin{aligned}
& N_{a}=\frac{1}{4}\left(1+\xi_{a}^{1} \xi^{1}\right)\left(1+\xi_{a}^{2} \xi^{2}\right), \\
& \xi_{a}^{1} \in[-1,1,1,-1], \quad \xi_{a}^{2} \in[-1,-1,1,1] .
\end{aligned}
$$

Eq. (46) can now be exactly verified for constant $S^{33}$ stress with respect to $\zeta$ coordinate, while from (47) it follows

$$
\underbrace{j_{0}\left(\int_{\square} \mathrm{nd} \xi^{1} \mathrm{~d} \xi^{2}\right)}_{0} \underbrace{\left(\int_{-1}^{1} \zeta \mathrm{d} \zeta\right)}_{0}=0 .
$$

The set of remaining equations in (45) is highly nonlinear and ought to be handled by an iterative procedure. If the Newton method is used for such a purpose, one employs constant linearization of (45). The latter can easily be carried out by symbolic manipulation (see [24]). Implementation of the theory presented above in this section can be done by replacing the energy functional governing the shell problem (42) with four functionals, which have the following forms when defined over the finite element domain:

$\Pi_{11}(\Phi)=\frac{1}{2} \int_{\square} \int_{-1}^{1} E_{i j} C^{i j k l} E_{k l} j \mathrm{~d} \zeta \mathrm{d} \xi^{1} \mathrm{~d} \xi^{2}-\Pi_{\mathrm{ext}}(\varphi)$,

$\Pi_{12}(\Phi, \boldsymbol{\alpha})=\frac{1}{2} \int_{\square} \int_{-1}^{1} E_{i j} C^{i j k l} \widetilde{E}_{k l} j \mathrm{~d} \zeta \mathrm{d} \xi^{1} \mathrm{~d} \xi^{2}$

$\Pi_{21}(\Phi, \boldsymbol{\alpha})=\frac{1}{2} \int_{\square} \int_{-1}^{1} \widetilde{E}_{i j} C^{i j k l} E_{k l} j \mathrm{~d} \zeta \mathrm{d} \xi^{1} \mathrm{~d} \xi^{2}$,

$\Pi_{22}(\boldsymbol{\alpha})=\frac{1}{2} \int_{\square} \int_{-1}^{1} \widetilde{E}_{i j} \mathrm{C}^{i j k l} \widetilde{E}_{k l} j \mathrm{~d} \zeta \mathrm{d} \xi^{1} \mathrm{~d} \xi^{2}$.

Variation and linearization of (53)-(55) carried out by symbolic manipulation with respect to the unknown quantities $\Phi=(\varphi$, a,$\lambda)$ and $\boldsymbol{\alpha}$ provide the following set of linear equations:

$$
\left\{\begin{array}{l}
\delta \Phi \\
\delta \boldsymbol{\alpha}
\end{array}\right\}^{\mathrm{T}}\left(\left[\begin{array}{cc}
\mathrm{K} & \widetilde{\mathrm{f}}^{\mathrm{T}} \\
\widetilde{\mathrm{f}} & \widetilde{\mathrm{H}}
\end{array}\right]\left\{\begin{array}{c}
\Delta \Phi \\
\Delta \boldsymbol{\alpha}
\end{array}\right\}=\left\{\begin{array}{l}
\widetilde{f} \\
\mathbf{0}
\end{array}\right\}-\left\{\begin{array}{c}
\widetilde{r} \\
\widetilde{h}
\end{array}\right\}\right),
$$

\footnotetext{
${ }^{7}$ In the following the procedures will be developed for $\alpha$ parameters although they are also valid for $\widehat{\alpha}$ parameters.
} 
where $\delta(\cdot)$ are admissible variations, $\Delta(\cdot)$ are linearized quantities, while matrices and vectors in (57) follow from the variation and linearization of (53)-(56). The subsequent solution procedure follows along with the lines traced by Ibrahimbegović and Wilson [16].

3.2. Incompatible modes based on a multiplicative decomposition of strains

An alternative manner to introduce the incompatible modes is at the level of an additive decomposition of the deformation gradient, which would result in the corresponding multiplicative decomposition of strains (e.g. see [17]). In the present case the incompatible modes choice is dictated by the goal to achieve a linear variation in the through-the-thickness direction.

We replace the base vector $a_{3}$ of the 6-parameter shell model - defined in the previous section in (34) - by a base vector $\overline{\mathrm{a}}_{3}$ of the following form:

$\overline{\mathrm{a}}_{3}\left(\xi^{1}, \xi^{2}, \zeta\right)=\underbrace{\mathrm{d}\left(\xi^{1}, \xi^{2}\right)}_{\mathrm{a}_{3}\left(\xi^{1}, \xi^{2}\right)}+\widetilde{\mathrm{b}}\left(\xi^{1}, \xi^{2}, \zeta\right)$,

where the vector $\widetilde{b}$ varies linearly in through-the-thickness direction such that

$\widetilde{\mathrm{b}}\left(\xi^{1}, \xi^{2}, \zeta\right)=\zeta \mathrm{b}\left(\xi^{1}, \xi^{2}\right)$.

Note that $b$ in (59) is still undefined. The enhancement (58) allows us to write the deformation gradient as

$$
\begin{aligned}
\overline{\mathrm{F}} & =\mathrm{a}_{\alpha} \otimes \mathrm{g}^{\alpha}+\overline{\mathrm{a}}_{3} \otimes \mathrm{g}^{3} \\
& =\left(\left[\frac{\partial \mathrm{x}}{\partial \xi}\right]+\widetilde{\mathrm{H}}\right)\left[\frac{\partial \mathrm{X}}{\partial \xi}\right]^{-1} \\
& =(\mathrm{J}+\widetilde{\mathrm{H}}) \mathrm{J}_{0}^{-1} \\
& =\mathrm{F}+\widetilde{\mathrm{F}}=\mathrm{a}_{i} \otimes \mathrm{g}^{i}+\widetilde{\mathrm{b}} \otimes \mathrm{g}^{3} .
\end{aligned}
$$

In (60) the natural coordinates are regrouped in a vector $\xi=\left\{\xi^{1}, \xi^{2}, \zeta\right\}^{\mathrm{T}}, \mathrm{F}$ is the deformation gradient of the 6 parameter theory described in the previous section and $\widetilde{F}$ is an enhanced part of the deformation gradient due to an enhancement of the base vector. To simplify the notation we collected in (60) the base vectors of deformed and initial configurations into the following matrices: ${ }^{8}$

$\mathrm{J}=\left[\mathrm{a}_{1}, \mathrm{a}_{2}, \mathrm{a}_{3}\right], \quad \widetilde{\mathrm{H}}=[0,0, \widetilde{\mathrm{b}}]$,

$\mathrm{J}_{0}=\left[\mathrm{g}_{1}, \mathrm{~g}_{2}, \mathrm{~g}_{3}\right], \quad \mathrm{J}_{0}^{-\mathrm{T}}=\left[\mathrm{g}^{1}, \mathrm{~g}^{2}, \mathrm{~g}^{3}\right]$.

Note that $a_{i}$ for the 6-parameter theory are defined in (34), while $g_{i}$ are given in (16). The right Cauchy-Green stretch tensor

\footnotetext{
${ }^{8}$ The usual simplifications carried out in the shell theory developments include also setting $\zeta=0$ when evaluating $\mathrm{J}_{0}$ (i.e. neglecting variation of metrics trough the shell thickness in the initial configuration), which is not done in the present work.
}

$\mathrm{C}=\overline{\mathrm{F}}^{\mathrm{T}} \overline{\mathrm{F}}=\mathrm{J}_{0}^{-\mathrm{T}}(\mathrm{J}+\widetilde{\mathrm{H}})^{\mathrm{T}}(\mathrm{J}+\widetilde{\mathrm{H}}) \mathrm{J}_{0}^{-1}$

leads to the Green-Lagrange strains in $\mathrm{g}^{i}$ base $\mathrm{E}=$ $\frac{1}{2}(\mathrm{C}-\mathbf{1})=E_{i j} \mathrm{~g}^{i} \otimes \mathrm{g}^{j}$ according to

$$
\begin{aligned}
E_{\alpha \beta} & =\frac{1}{2}\left(a_{\alpha} \cdot a_{\beta}-g_{\alpha} \cdot g_{\beta}\right), \\
E_{\alpha 3} & =\frac{1}{2}[a_{\alpha} \cdot(d+\widetilde{b})-\underbrace{g_{\alpha} \cdot g_{3}}_{0}]=\frac{1}{2} a_{\alpha} \cdot d+\frac{1}{2} a_{\alpha} \cdot \widetilde{b}, \\
E_{33} & =\frac{1}{2}\left((d+\widetilde{b}) \cdot(d+\widetilde{b})-g_{3} \cdot g_{3}\right] \\
& =\frac{1}{2}\left(d \cdot d-g_{3} \cdot g_{3}\right)+\frac{1}{2}(2 d \cdot \widetilde{b}+\widetilde{b} \cdot \widetilde{b}) .
\end{aligned}
$$

We can conclude from (63) that

$E_{i j}=H_{i j}+\zeta K_{i j}+\zeta^{2} L_{i j}$,

where $H_{i j}, K_{\alpha \beta}$ and $L_{\alpha \beta}$ strains are the same as those already given in OO()(37)-(39), respectively, while other strains of the 6-parameter model from Section 3.1 are modified to be

$$
\begin{aligned}
K_{\alpha 3} & =\frac{1}{2}\left(\mathrm{~d}_{, \alpha} \cdot \mathrm{d}+\boldsymbol{\varphi}_{, \alpha} \cdot \mathrm{b}\right), \\
K_{33} & =\frac{1}{2}(2 \mathrm{~d} \cdot \mathrm{b}), \\
L_{\alpha 3} & =\frac{1}{2}\left(\mathrm{~d}_{, \alpha} \cdot \mathrm{b}\right), \\
L_{33} & =\frac{1}{2}(\mathrm{~b} \cdot \mathrm{b}) .
\end{aligned}
$$

Through-the-thickness variation of all strains is quadratic.

Note that one cannot use any more an additive split of the total strain, as in the previously described implementation. However, the admissible variations of the Green-Lagrange strains (64) can still be written in terms of an additive decomposition as

$\delta E_{i j}+\delta \widetilde{E}_{i j}$

where

$\delta E_{i j}=\frac{1}{2}\left(\delta a_{i} \cdot a_{j}+a_{i} \cdot \delta a_{j}\right)$

and

$\delta \widetilde{E}_{i j}= \begin{cases}0, & i, j=1 \text { or } 2, \\ \frac{1}{2}\left(\delta \mathrm{a}_{\alpha} \cdot \widetilde{\mathrm{b}}+\mathrm{a}_{\alpha} \cdot \delta \widetilde{\mathrm{b}}\right), & i \text { or } j=3, \\ \delta \mathrm{d} \cdot \widetilde{\mathrm{b}}+\delta \widetilde{\mathrm{b}} \cdot \mathrm{d}+\delta \widetilde{\mathrm{b}} \cdot \widetilde{\mathrm{b}}, & i, j=3 .\end{cases}$

The corresponding variational formulation can be obtained by generalizing the incompatible mode method of Ibrahimbegović and Frey [19] from membranes to shells. To that end, two equations governing equilibrium can be written as

$$
\begin{aligned}
D \Pi(\underbrace{\varphi, \mathrm{a}, \lambda}_{\Phi}, \widetilde{H}_{i}^{j}, P_{i}^{j}) \cdot \delta \Phi \\
=\int_{A} \int_{h_{0}} \delta E_{i j} S^{i j} \mathrm{~d} V-D \Pi_{\mathrm{ext}}(\varphi) \cdot \delta \varphi=0,
\end{aligned}
$$


for the corresponding variation of the compatible displacement field and

$D \Pi\left(\Phi, \widetilde{H}_{i}^{j}, P_{i}^{j}\right) \cdot \delta \widetilde{H}_{i}^{j}=\int_{A} \int_{h_{0}} \delta \widetilde{E}_{i j} S^{i j} \mathrm{~d} V=0$,

for the incompatible mode variations. In (70) and (71) we compute the second Piola-Kirchhoff stress from the constitutive equations for the first Piola-Kirchhoff stress, $P_{i}^{j}=\partial W / \partial F_{i}^{j}$, along with the geometric transformation connecting the two kinds of Piola-Kirchhoff stresses, $\mathrm{S}=\mathrm{F}^{-1} \mathrm{P}$. The last two equations have to be accompanied by an additional expression which guaranties the convergence of the incompatible mode method in the sense of the patch test, which can be written as

$\int_{A} \int_{h_{0}} \widetilde{H}_{i}^{j} \mathrm{~d} V=0$

In the finite element implementation we choose $b$, see (59), to be

$\mathrm{b}=A_{33} \mathrm{~d}$,

where $d$ is the extensible shell director already expressed in (10). From (58) and (59) follows that the enhanced base vector at the deformed configuration is of the form

$\overline{\mathrm{a}}_{3}=\left(1+\zeta A_{33}\right) \mathrm{d}$.

An interpolation of $A_{33}$ over the finite element may be chosen again either by (48) or by standard bi-linear interpolation functions (49). With this choice of interpolation, the patch test is naturally satisfied, which can be proved in the same manner as already shown in (47) and (52).

\section{Interpolation and FE implementation}

Finite element approximation of the shell models developed in the above sections is based on finite elements with four nodes on the middle surface. Convective coordinates $\xi^{1}$ and $\xi^{2}$ from previous sections are now replaced with isoparametric coordinates of a shell finite element. According to the isoparametric concept we use standard bi-linear interpolation functions to define middle surface geometry within one element as

$\boldsymbol{\varphi}_{0}=\sum_{a=1}^{n_{\mathrm{en}}} N_{a}\left(\xi^{1}, \xi^{2}\right)\left(\boldsymbol{\varphi}_{0}\right)_{a}, \quad \boldsymbol{\varphi}=\sum_{a=1}^{n_{\mathrm{en}}} N_{a}\left(\xi^{1}, \xi^{2}\right) \boldsymbol{\varphi}_{a}$,

where the number of element nodes $n_{\mathrm{en}}=4, N_{a}: \square \rightarrow \mathbb{R}$ are the corresponding shape functions already given in (51), whereas $(\cdot)_{a}$ are the corresponding nodal values. Through-the-thickness kinematic variables are interpolated in the same manner $\widetilde{\lambda}=\sum_{a=1}^{n_{\mathrm{en}}} N_{a} \tilde{\lambda}_{a}, \quad \widetilde{\lambda}=1-\lambda$,

$q=\sum_{a=1}^{n_{\mathrm{en}}} N_{a} q_{a}$

It is indicated in (76) that rather than the thicknesschange variable $\lambda$ we interpolate $\widetilde{\lambda}$ in order to have zero values of all unknown kinematic variables at the initial configuration. Current thickness is then expressed as

$h=h_{0}(1-\tilde{\lambda}), \quad \tilde{\lambda} \leqslant 1$.

Approximation of the shell director requires special attention in order to obtain good numerical performance of the 6- and the 7-parameter models for very thin shells. Very often parasitic through-the-thickness strains are induced through a simple interpolation of the shell director, especially in formulations where rotations are avoided by introducing the so-called difference vector (e.g. [13,29]). In some works the effect of artificial thickness strains is avoided by assumed strain approximation of $E_{33}$ (e.g. [5]). To avoid this approximation in the present work, the shell director is normalized over an element in order to always remain exactly of a unit length at the integration points

$\mathrm{a}=\frac{\widehat{\mathrm{a}}}{\|\widehat{\mathrm{a}}\|}, \quad \widehat{\mathrm{a}}=\sum_{a=1}^{n_{\mathrm{en}}} N_{a} \mathrm{a}_{a}$,

$\mathrm{g}=\frac{\widehat{\mathrm{g}}}{\|\widehat{\mathrm{g}}\|}, \quad \widehat{\mathrm{g}}=\sum_{a=1}^{n_{\mathrm{en}}} N_{a} \mathrm{~g}_{a}$

The nodal shell director in a deformed configuration is given as a function of the total nodal material rotation vector $\boldsymbol{\vartheta}_{a}=\left\{\vartheta_{a}^{1}, \vartheta_{a}^{2}\right\}^{\mathrm{T}}$ with (see [10])

$\mathrm{a}_{a}=\Lambda_{0 a}\left(\cos \vartheta_{a}\{0,0,1\}^{\mathrm{T}}+\frac{\sin \vartheta_{a}}{\vartheta_{a}}\left\{\vartheta_{a}^{2},-\vartheta_{a}^{1}, 0\right\}^{\mathrm{T}}\right)$

where $\vartheta_{a}=\left\|\vartheta_{a}\right\|$ is the Euclidean norm of the nodal rotation vector and $\Lambda_{0 a}=\left[\mathrm{e}_{1 a}, \mathrm{e}_{2 a}, \mathrm{~g}_{a}\right]$ is the initial nodal rotation matrix providing orientation for the nodal rotation parameters $\vartheta_{a}^{1}$ and $\vartheta_{a}^{2}$. Vectors $\mathrm{e}_{1 a}, \mathrm{e}_{2 a}$ and $\mathrm{g}_{a}$ define Cartesian basis at node $a$.

The virtual and the incremental quantities are interpolated as

$$
\begin{aligned}
& \delta \boldsymbol{\varphi}=\sum_{a=1}^{n_{\mathrm{en}}} N_{a} \delta \boldsymbol{\varphi}_{a}, \quad \delta \widetilde{\lambda}=\sum_{a=1}^{n_{\mathrm{en}}} N_{a} \delta \widetilde{\lambda}_{a}, \\
& \delta q=\sum_{a=1}^{n_{\mathrm{en}}} N_{a} \delta q_{a},
\end{aligned}
$$


$\delta \mathrm{a}=\frac{1}{\|\widehat{a}\|}(\mathrm{I}-\mathrm{a} \otimes \mathrm{a}) \delta \widehat{\mathrm{a}}, \quad \delta \widehat{\mathrm{a}}=\sum_{a=1}^{n_{\mathrm{en}}} N_{a} \delta \mathrm{a}_{a}$.

Derivatives of the interpolated quantities with respect to $\xi^{\alpha}$ coordinates can be obtained trivially except for $\mathrm{a}_{, \alpha}$ and $\delta a_{, \alpha}$. Linearized quantities $\Delta \delta a$ and $\Delta \delta a_{, \alpha}$ have even more complicated forms and can be mainly obtained by using symbolic manipulations.

In order to eliminate the shear locking effect, the transverse shear strains are interpolated over a parent element by using the assumed natural strain (ANS) concept of Bathe and Dvorkin [4] according to

$$
\begin{aligned}
& \widehat{E}_{13}=\frac{1}{2}\left[\left(1-\xi^{2}\right) E_{13}^{B}+\left(1+\xi^{2}\right) E_{13}^{D}\right], \\
& \widehat{E}_{23}=\frac{1}{2}\left[\left(1-\xi^{1}\right) E_{23}^{A}+\left(1+\xi^{1}\right) E_{23}^{C}\right] .
\end{aligned}
$$

Strains $E_{i 3}^{(\cdot)}$ are evaluated at the mid-side point $(\cdot)$ in accordance with the expressions derived in the previous sections. Linear and higher-order terms of $E_{i 3}^{(\cdot)}$ are automatically neglected by choosing the shear interpolation points $A, B, C$ and $D$ on the middle surface of the shell finite element corresponding to $\zeta=0$. Positions of those points are $\varphi_{0}^{L}=\frac{1}{2}\left[\left(\varphi_{0}\right)_{M}+\left(\varphi_{0}\right)_{N}\right]$, where $(L, M$, $N) \in\{(A, 1,2),(B, 2,3),(C, 3,4),(D, 1,4)\}$. Finite element approximation of the transverse shear strains across the thickness of the shell element is therefore assumed to be constant.

Numerical integration is performed at $2 \times 2 \times 2$ Gauss integration points. At each integration point a local Cartesian basis $e_{i}$ is introduced in such a way that the third base vector is identical to the initial shell director and the other two are perpendicular to it

$e_{3}=g, \quad e_{1} \perp e_{2}, \quad e_{1} \times E_{2}=E_{3}$.

Having defined the current and the initial position vectors over a finite element domain, we may obtain the deformation gradient at an integration point as

$\mathrm{F}=\frac{\partial \mathrm{x}}{\partial \mathrm{X}}=\left[\frac{\partial \mathrm{x}}{\partial \xi}\right]\left[\frac{\partial \mathrm{X}}{\partial \xi}\right]^{-1}=\mathrm{JJ}_{0}^{-1}$,

where $\mathrm{J}$ and $\mathrm{J}_{0}$ are defined in (61). The right CauchyGreen stretch tensor can be computed from the deformation gradient ${ }^{9}$ as

$\mathrm{C}=\mathrm{F}^{\mathrm{T}} \mathrm{F}=\mathrm{J}_{0}^{-\mathrm{T}}\left(\mathrm{J}^{\mathrm{T}} \mathrm{J}\right) \mathrm{J}_{0}^{-1}=C_{i j} \mathrm{~g}^{i} \otimes \mathrm{g}^{j}$,

where the corresponding components in the $\mathrm{g}^{i}$ basis are $C_{i j}=\mathrm{a}_{i} \cdot \mathrm{a}_{j}=\mathrm{J}^{\mathrm{T}} \mathrm{J}$. The transformation of $C_{i j}$ components to the $C_{i j}^{*}$ components, which are defined with respect to the $e_{i}$ basis (85), can be performed according to

\footnotetext{
${ }^{9}$ We note again that the deformation gradient is enriched for the method described in Section 3.2, see (60). In that case we use Eq. (62) instead of Eq. (87)
}

$\left[C_{i j}^{*}\right]=\left[\mathrm{e}_{1}, \mathrm{e}_{2}, \mathrm{~g}\right]^{\mathrm{T}} \mathrm{J}_{0}^{-\mathrm{T}}\left[C_{i j}\right] \mathrm{J}_{0}^{-1}\left[\mathrm{e}_{1}, \mathrm{e}_{2}, \mathrm{~g}\right]=\mathrm{T}\left[C_{i j}\right] \mathrm{T}^{\mathrm{T}}$,

where the transformation matrix has the following form:

$\mathrm{T}=\left[\begin{array}{ccc}\mathrm{g}^{1} \cdot \mathrm{e}_{1} & \mathrm{~g}^{2} \cdot \mathrm{e}_{1} & 0 \\ \mathrm{~g}^{1} \cdot \mathrm{e}_{2} & \mathrm{~g}^{2} \cdot \mathrm{e}_{2} & 0 \\ 0 & 0 & 2 / h_{0}\end{array}\right]$

Strains in the local Cartesian frame (85) can then be calculated as

$E_{i j}^{*}=\frac{1}{2}\left(C_{i j}^{*}-\delta_{i j}\right)$.

The transformation of the transverse shear strains from the $\xi$ coordinates to the local Cartesian coordinates defined with basis $e_{i}(85)$ is performed as (e.g. see $\left.[9,18]\right)$

$\left\{\begin{array}{l}E_{13}^{*} \\ E_{23}^{*}\end{array}\right\}=\left[\begin{array}{ll}g_{1} \cdot e_{1} & g_{1} \cdot e_{2} \\ g_{2} \cdot e_{1} & g_{2} \cdot e_{2}\end{array}\right]^{-1} \frac{2}{h_{0}}\left\{\begin{array}{l}\widehat{E}_{13} \\ \widehat{E}_{23}\end{array}\right\}$,

where an additional term $2 / h_{0}$ appears due to the definition of $\zeta$ coordinate which leads to the base vectors $\mathrm{g}_{3}=\left(h_{0} / 2\right) \mathrm{g}$ and $\mathrm{g}^{3}=\left(2 / h_{0}\right) \mathrm{g}$.

With (90) and (91) we can define the potential energy of the shell in terms of $E_{i j}^{*}$ strains. Symbolic manipulation (see $[22,24]$ ) is further used to obtain its first and second derivative with respect to the nodal unknown kinematic variables leading to residuals and stiffness matrix. When internal variables are present, the final form of the stiffness matrix is obtained by the procedure of static condensation.

\section{Numerical examples}

In this section we present some results of numerical simulations. The computations were carried out by a research version of the computer program AceGen (see [23]). We have implemented 4-noded finite elements listed in Table 1: five for the SVK material and four for the $\mathrm{NH}$ material with a strain energy function of the form (25). For the 5-parameter SVK element we condensed 3d constitutive relations by using the condition $S^{33}=0$. In all examples we used $2 \times 2 \times 2$ Gaussian integration rule. A tolerance of $10^{-9}$ for the Euclidean norm of iterative nodal values was employed in the Newton iteration scheme for each of the examples. In Appendices A and $\mathrm{B}$ we present an input for symbolic manipulations using AceGen program which produce the $7 E$ element.

\subsection{Bending of cantilever beam by end force}

This example demonstrates the ability of finite elements based on non-standard theories to recover $2 \mathrm{~d}$ shell behavior in the thin shell limit. It was considered e.g. by Simo et al. [29], Büchter et al. [13], Parisch [27], Abbasi and Meguid [1]; however, the data in those 
Table 1

Finite elements used in numerical examples

\begin{tabular}{ll}
\hline $5 E$ & 5-parameter model with exact $(E)$ strains \\
$7 E$ & 7-parameter model with exact $(E)$ strains; see $(17)$ \\
$7 R$ & 7-parameter model with reduced $(R)$ strains; see (23) \\
$6 E A$ & 6-parameter model with exact $(E)$ strains and \\
& $\begin{array}{l}\text { incompatible mode based on an additive }(A) \\
\text { decomposition of strains; interpolation (48) }\end{array}$ \\
$6 E M$ & $\begin{array}{l}\text { 6-parameter model with exact }(E) \text { strains and } \\
\text { incompatible mode based on a multiplicative }(M) \\
\text { decomposition of strains; interpolation }(48)\end{array}$ \\
\hline
\end{tabular}

references vary. We consider a beam of length $L=10$, width $B=1$ and thickness $h_{0}$, which is clamped at one end and subjected to two point forces $F=F_{1}=$ $40 \times 10^{3} \times h_{0}^{3}$ acting on the middle surface of the free end (see Fig. 1). Four different values $(2,1,0.1,0.01)$ are used for thickness producing length-to-thickness ratios to be 5, 10, 100 and 1000 . Material parameters are $E=10 \times 10^{6}, v=0.3$. A mesh of $10 \times 1$ element is used. We present results of the nonlinear analyses. Beam end deflections produced by different elements are given in Tables 2 and 3. In order to compare our results with those obtained by Simo et al. [29] (who used 5-parameter stress resultant element and 6-parameter stress resultant element with plain stress formulation for the bending part of constitutive equations) we also present beam end deflections for $F=F_{2}=50 \times 10^{3} \times h_{0}^{3}$ in Table 4. It can be observed from Tables 2-4 that in the thin shell limit $\left(L / h_{0}=1000\right)$ the elements $5 E, 6 A D$ and $6 M D$ produce identical results, which indicates that formulations based on incompatible modes recover the thin shell solution, while elements based on the 7-parameter theory ( $7 E$ and $7 R$ ) produce approximately $99 \%$ of the $5 E$ element results. It can be also observed that the difference between the $7 E$ and the $7 R$ solutions increases with decreasing $L / h_{0}$ ratio, which suggests that the higher-order strain terms are more important for thicker shells. The same is valid for incompatible mode formulations: the difference in results increases with increase of thickness. In Table 5

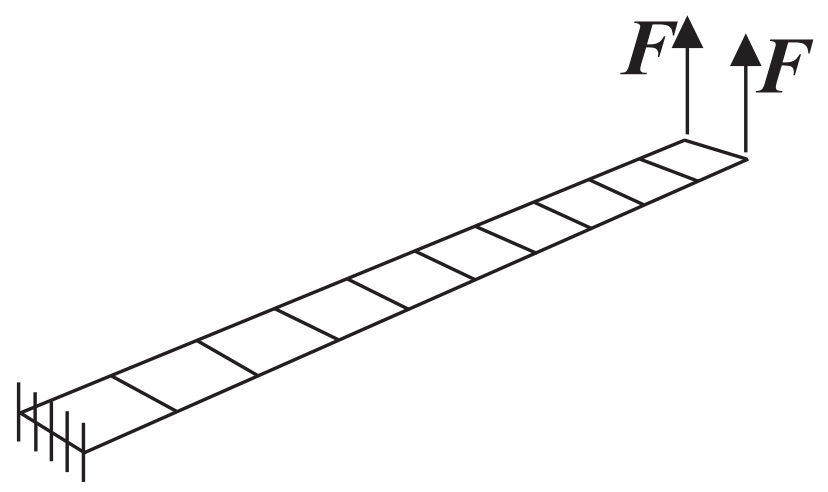

Fig. 1. Bending of beam by end force: mesh and loading.
Table 2

Bending of beam by end force; end deflection at $F=F_{1}$

\begin{tabular}{|c|c|c|c|c|}
\hline \multirow[t]{2}{*}{ Element } & \multicolumn{4}{|l|}{$L / h_{0}$} \\
\hline & 5 & 10 & 100 & 1000 \\
\hline \multicolumn{5}{|c|}{ SVK material } \\
\hline $5 E$ & 7.3492 & 7.1188 & 7.0477 & 7.0470 \\
\hline $7 E$ & 7.3037 & 7.0568 & 6.9869 & 6.9874 \\
\hline $7 R$ & 7.2624 & 7.0453 & 6.9868 & 6.9874 \\
\hline $6 E A$ & 7.3797 & 7.1278 & 7.0477 & 7.0470 \\
\hline $6 E M$ & 7.3867 & 7.1294 & 7.0478 & 7.0470 \\
\hline
\end{tabular}

Table 3

Bending of beam by end force; end deflection at $F=F_{1}$

\begin{tabular}{lllll}
\hline Element & $L / h_{0}$ & & & \\
\cline { 2 - 5 } & 5 & 10 & 100 & 1000 \\
\hline NH material & & & & \\
$7 E$ & 7.3404 & 7.0691 & 6.9870 & 6.9874 \\
$7 R$ & 7.3083 & 7.0586 & 6.9869 & 6.9874 \\
$6 E A$ & 7.4146 & 7.1401 & 7.0479 & 7.0467 \\
$6 E M$ & 7.4184 & 7.1411 & 7.0479 & 7.0467 \\
\hline
\end{tabular}

Table 4

Bending of beam by end force; end deflection at $F=F_{2}$

\begin{tabular}{lll}
\hline Element & $L / h_{0}$ & \\
\cline { 2 - 3 } & 10 & 1000 \\
\hline SVK material & 7.5178 & 7.4331 \\
$5 E$ & 7.4560 & 7.3751 \\
$7 E$ & 7.4435 & 7.3751 \\
$7 R$ & 7.5276 & 7.4331 \\
$6 E A$ & 7.5294 & 7.4331 \\
$6 E M$ & 7.3839 & 7.3053 \\
$7 R$-condensed, Ref. [29] & 7.3849 & 7.3053 \\
$5 R$, Ref. [29] &
\end{tabular}

Table 5

Bending of beam by end force; maximum values of $\tilde{\lambda}$ (at the point of maximum curvature) multiplied by 100 at $F=F_{1}$

\begin{tabular}{llll}
\hline Element & $L / h_{0}$ & & \\
\cline { 2 - 4 } & 5 & 10 & 1000 \\
\hline$S V K$ material & & & \\
$7 E$ & 2.847 & 0.580 & 0.005 \\
$7 R$ & 3.626 & 0.790 & 0.007 \\
$6 E A$ & 3.154 & 0.772 & 0.008 \\
$6 E M$ & 2.389 & 0.804 & 0.008 \\
\hline
\end{tabular}

we collect maximum values of $\tilde{\lambda}$ at the points with maximum curvatures. Those values are small: maximum thickness change is around 3\% for beams with $L / h_{0}=5$. 
Distribution of $\tilde{\lambda}$ through the beam length is shown in Fig. 2, and a sequence of deformed configurations is shown in Fig. 3. Table 6 presents the total number of iterations when the forces $F=F_{1}$ are applied in five equal increments. It is interesting to note that the number of iterations depends only on the length-tothickness ratio.

\subsection{Cylinder under line load}

This example was considered by Büchter et al. [13] to test the behavior of $3 \mathrm{~d}$ shell formulations for thin and thick shells. A cylinder of length $L=30 \mathrm{~cm}$, radius $R=9 \mathrm{~cm}$ and thickness $h_{0}=(0.2 \mathrm{~cm}, 2 \mathrm{~cm})$ is supported and subjected to a line load $p$ as shown in Fig. 4. Due to symmetry conditions only one-quarter of the cylinder is discretized by $16 \times 64$-node finite elements. The parameters of the $\mathrm{NH}$ material are $\mu=6000 \mathrm{kN} / \mathrm{cm}^{2}$, $\lambda=24,000 \mathrm{kN} / \mathrm{cm}^{2}$ or $E=16,800 \mathrm{kN} / \mathrm{cm}^{2}, v=0.4$. The load, which is acting on the middle surface, was applied in five equal steps. In Table 7 we compare our results with those given by Büchter et al. [13] who used a mesh of $16 \times 68$-node elements with $2 \times 2 \times 3$ Gauss integration points. Comparison is carried out for a total load when the displacement of the point under the force at the free edge (point A) equals $16 \mathrm{~cm}$. The response

Min: 0. Max, 00362622

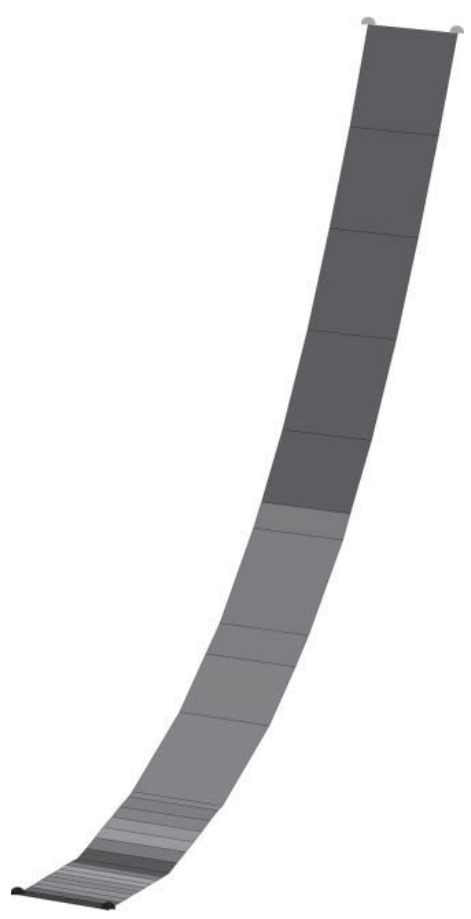

Fig. 2. Bending of beam by end force: distribution of $\tilde{\lambda}$ throughout the beam when $F=F_{1}$; SVK material; $7 R$ element; $L / h_{0}=5$.

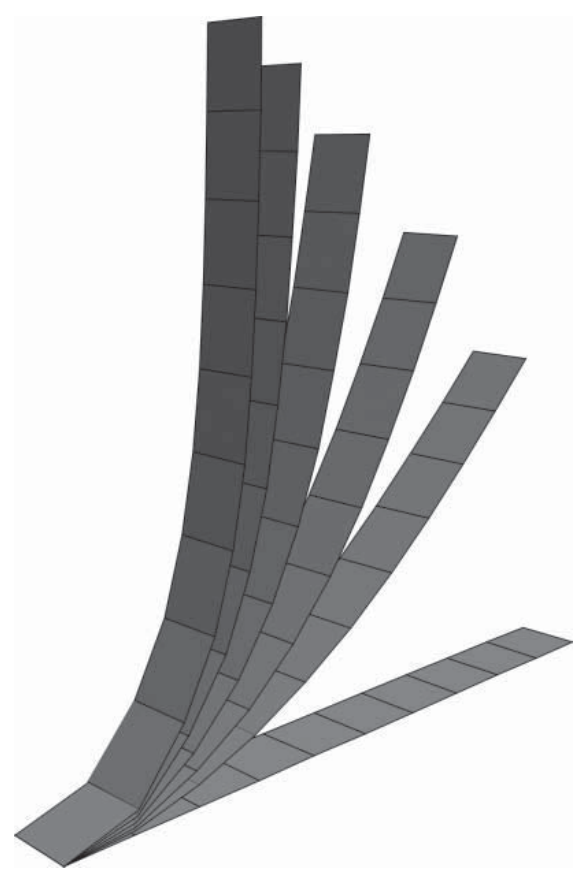

Fig. 3. Bending of beam by end force: sequence of deformed meshes; final configuration corresponds to $F=4 F_{1}$; NH material; $7 R$ element; $L / h_{0}=2$.

Table 6

Bending of beam by end force; total number of iterations; $F=F_{1}$

\begin{tabular}{lllll}
\hline Element & $L / h_{0}$ & & & \\
\cline { 2 - 5 } & 5 & 10 & 100 & 1000 \\
\hline SVK material & & & & \\
$5 E$ & 34 & 37 & 50 & 62 \\
$7 E$ & 34 & 37 & 50 & 63 \\
$7 R$ & 34 & 37 & 50 & 63 \\
$6 E A$ & 34 & 37 & 51 & 63 \\
$6 E M$ & 34 & 37 & 51 & 63 \\
\hline
\end{tabular}

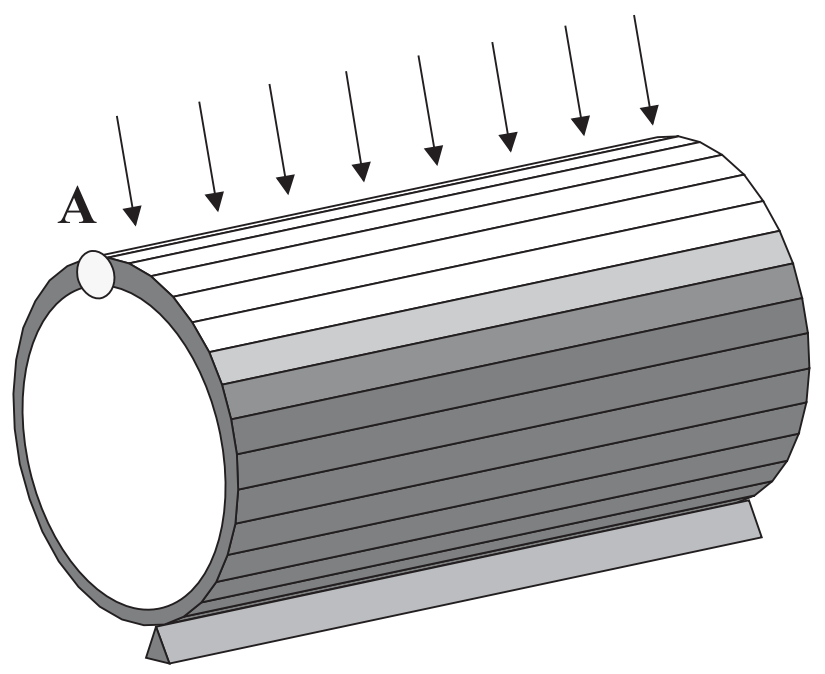

Fig. 4. Hyperelastic cylinder: support and loading conditions. 
Table 7

Hyperelastic cylinder; total load $[\mathrm{kN}]$ when displacement of point $\mathrm{A}$ is $16 \mathrm{~cm} ; 16 \times 6$ elements

\begin{tabular}{lll}
\hline Element, Material & \multicolumn{2}{l}{$h_{0}$} \\
\cline { 2 - 3 } & $0.2 \mathrm{~cm}$ & $2 \mathrm{~cm}$ \\
\hline $5 E, \mathrm{SVK}$ & 35.13 & 28961 \\
$7 E, \mathrm{SVK}$ & 35.43 & 28561 \\
$7 R, \mathrm{SVK}$ & 35.46 & 30027 \\
$6 E A, \mathrm{SVK}$ & 35.12 & 28935 \\
$6 E M, \mathrm{SVK}$ & 35.13 & 28706 \\
$7 E, \mathrm{NH}$ & 35.47 & 29445 \\
$7 R, \mathrm{NH}$ & 35.49 & 30805 \\
$6 E A, \mathrm{NH}$ & 35.12 & 29731 \\
$6 E M, \mathrm{NH}$ & 35.13 & 29530 \\
$5 E, \mathrm{NH}, \mathrm{Ref} .[13]$ & 34.71 & 28636 \\
$5 R, \mathrm{NH}, \mathrm{Ref} .[13]$ & 34.70 & 28428 \\
$6 E A, \mathrm{NH}, \mathrm{Ref} .[13]$ & 34.71 & 29984 \\
$6 R A, \mathrm{NH}, \mathrm{Ref} .[13]$ & 34.87 & 33680 \\
\hline
\end{tabular}

curves for all elements are very similar. This can be seen from Fig. 5 where the total force $P=p * L$ is plotted versus the displacement of point $A$. In Fig. 6 a sequence of deformed meshes is presented and in Fig. 7 the distribution of thickness change is plotted. Maximum values of about $4 \%$ are at the free end of the shell (at the region of maximum curvature) and at the point $A$. The thickness stretch of the point $A$ with respect to the total load is presented in Figs. 8 and 9. We note that the thickness at the first two increments was bigger than the initial (i.e. $\widetilde{\lambda}<0$ ). Evolution of $q$ variable at the point $A$ is given in Fig. 10.

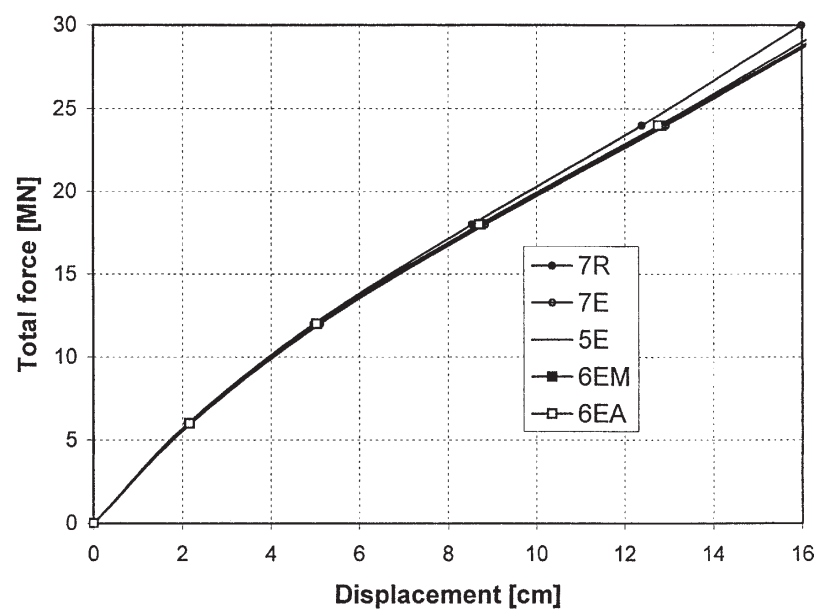

Fig. 5. Hyperelastic cylinder: load versus displacement curves; SVK material; $h_{0}=2 \mathrm{~cm}$.

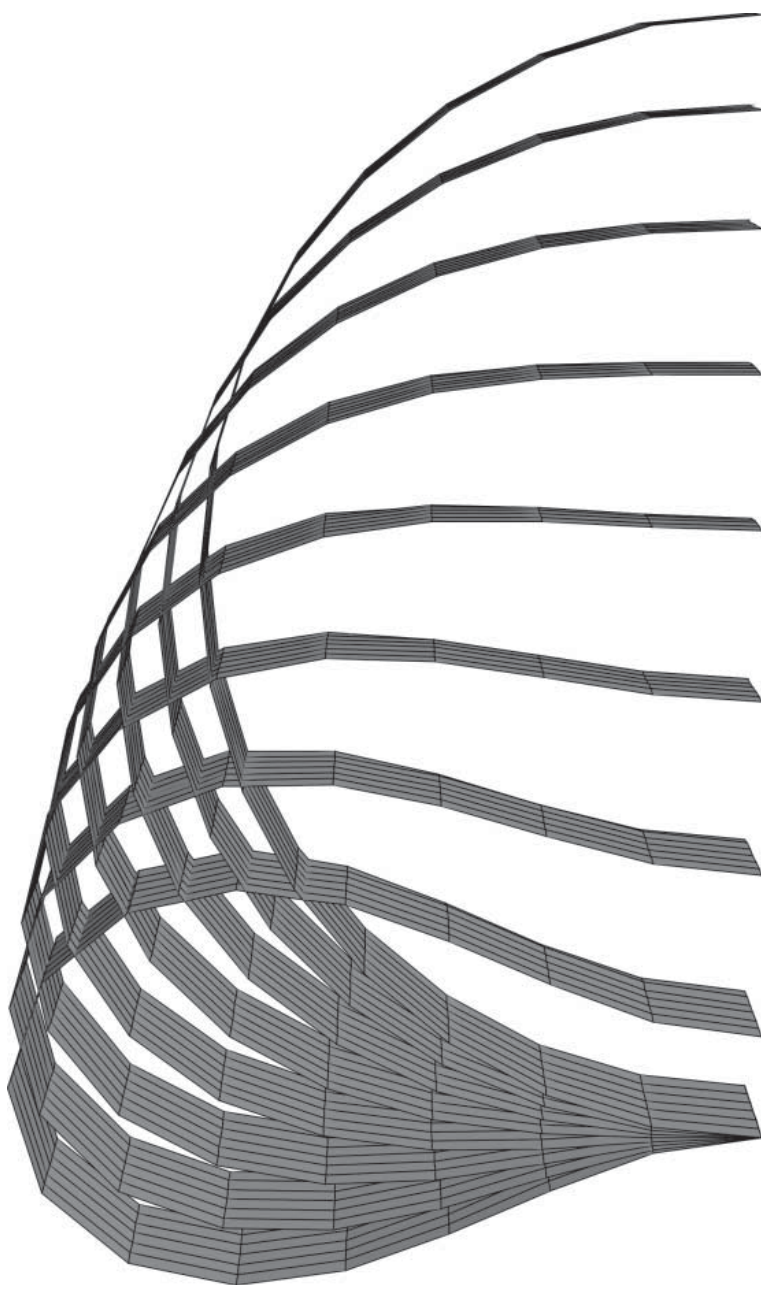

Fig. 6. Hyperelastic cylinder: a sequence of deformed configurations.

\subsection{Clamped sphere under point load}

The collapse of a thick clamped hemispherical shell was analyzed in many papers. Among them we mention Simo and co-workers $[29,30]$ who also used elements capable to account for through-the-thickness stretching, and Eberlein and Wriggers [14] who used axisymmetrical quasi-Kirchhoff element and compared its solution with many others. With this problem we demonstrate the very localized effect of the thickness change under concentrated force. The shell with radius $R=26.3 \mathrm{~mm}$ and thickness $h_{0}=4.4 \mathrm{~mm}$ is clamped along the circumference and subjected to a point load at the pole (Fig. 11). The load is acting on the shell middle surface. Shell was analyzed for the SVK material with the following properties: $E=4 \times 10^{3} \mathrm{kN} / \mathrm{m}^{2}$ and $v=0.49$. Due to symmetry conditions only one-quarter was analyzed by using a mesh of $16 \times 16$ elements. Displacement control was used to drive the top (middle surface) point of the hemisphere to the distance equal to the radius. 10 time- 

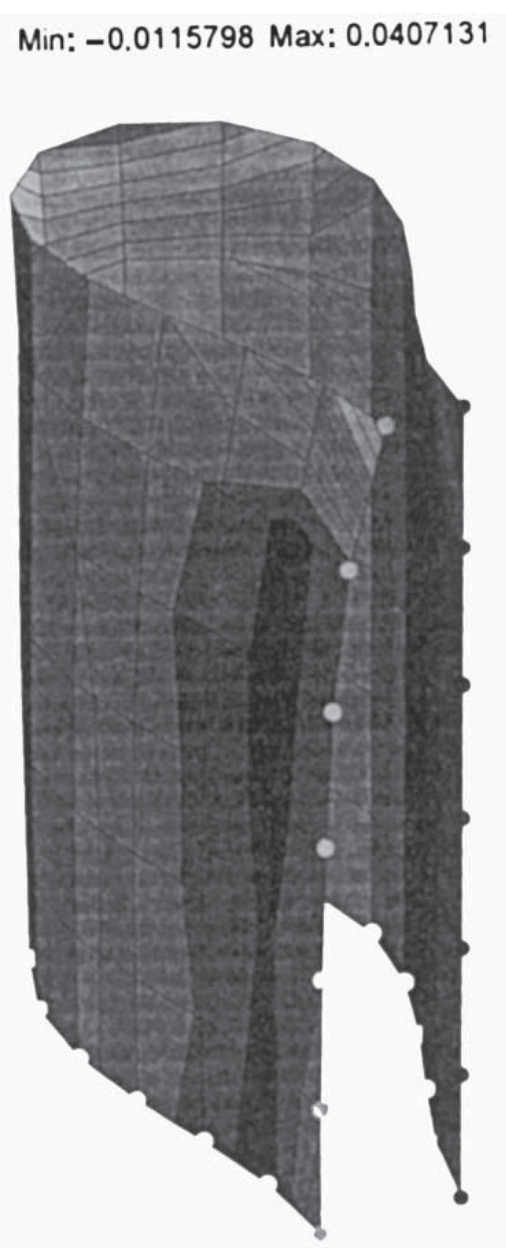

Fig. 7. Hyperelastic cylinder: distribution of thickness change $\tilde{\lambda}$ at total force $P=4 \times 7500 \mathrm{kN}$; SVK material; $6 E A$ element.

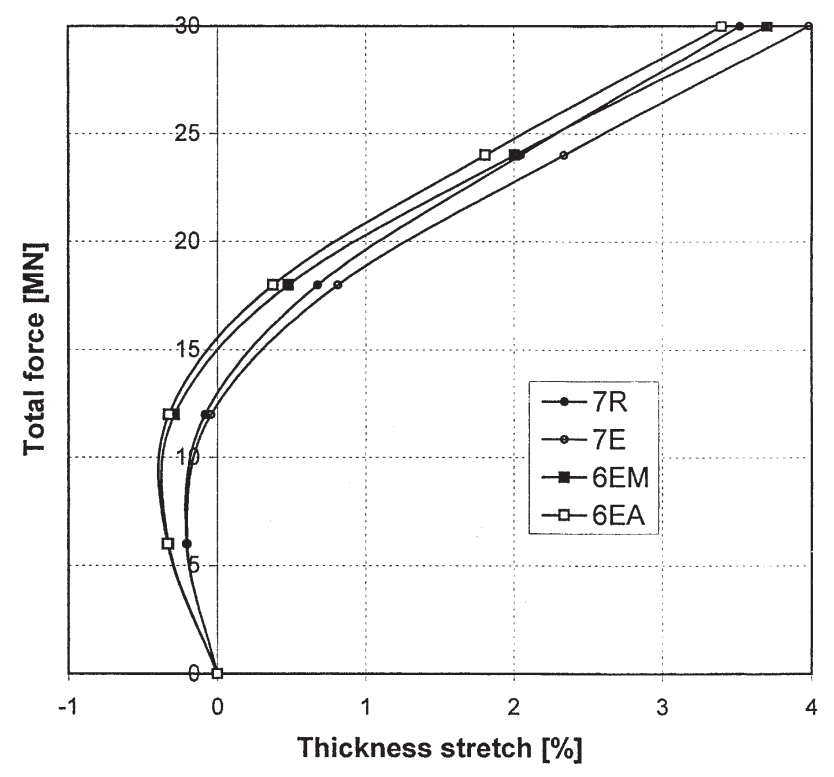

Fig. 8. Hyperelastic cylinder: thickness stretch $\tilde{\lambda}$ at point $A$ versus total force; SVK material; $h_{0}=2 \mathrm{~cm}$.

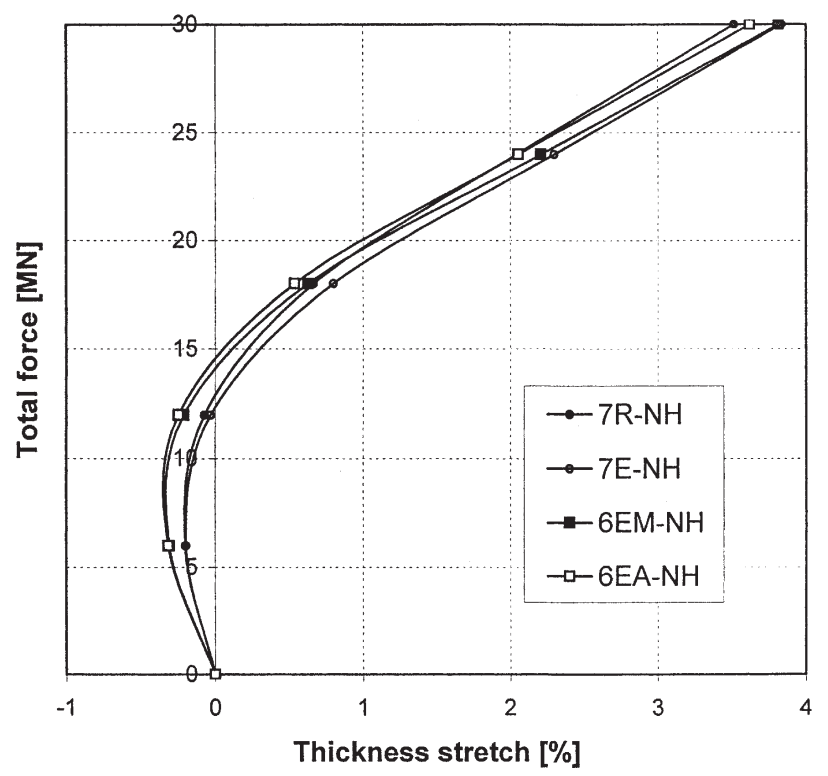

Fig. 9. Hyperelastic cylinder: thickness stretch $\tilde{\lambda}$ at point $A$ versus total force; NH material; $h_{0}=2 \mathrm{~cm}$.

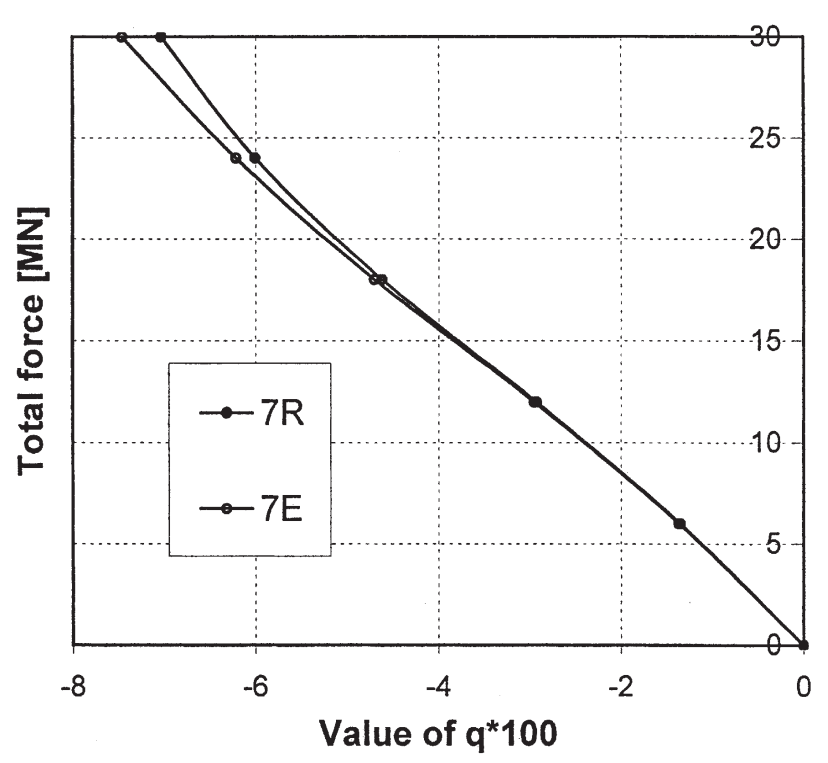

Fig. 10. Hyperelastic cylinder: values of $q$ at point $A$ versus total force; NH material; $h_{0}=2 \mathrm{~cm}$.

steps were used for the $5 E$ element and 20 for all other elements. Load versus deflection curves are presented in Fig. 12 for the 7-parameter elements and in Fig. 13 for the 6-parameter elements. All elements reached the final configuration except the $6 E M$ which diverged at $u / R=$ 0.75 , while the $7 E$ element produced thickness change $\tilde{\lambda}>1$ (i.e. negative thickness) for $u / R>0.8$. "Axy-mid" in Figs. 12 and 13 denotes curves obtained by a mesh of $100 \times 20$ axisymmetrical elements (see Fig. 14) of 


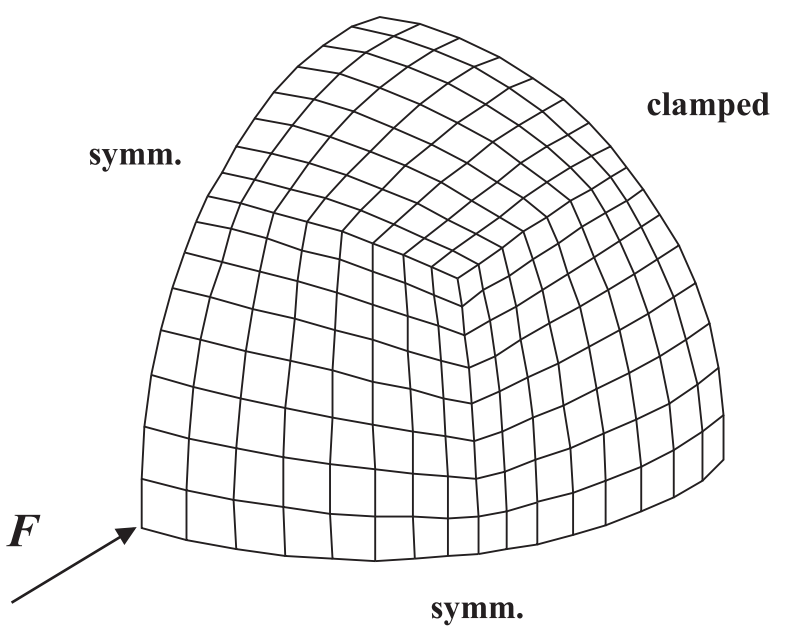

Fig. 11. Clamped sphere: mesh and loading.

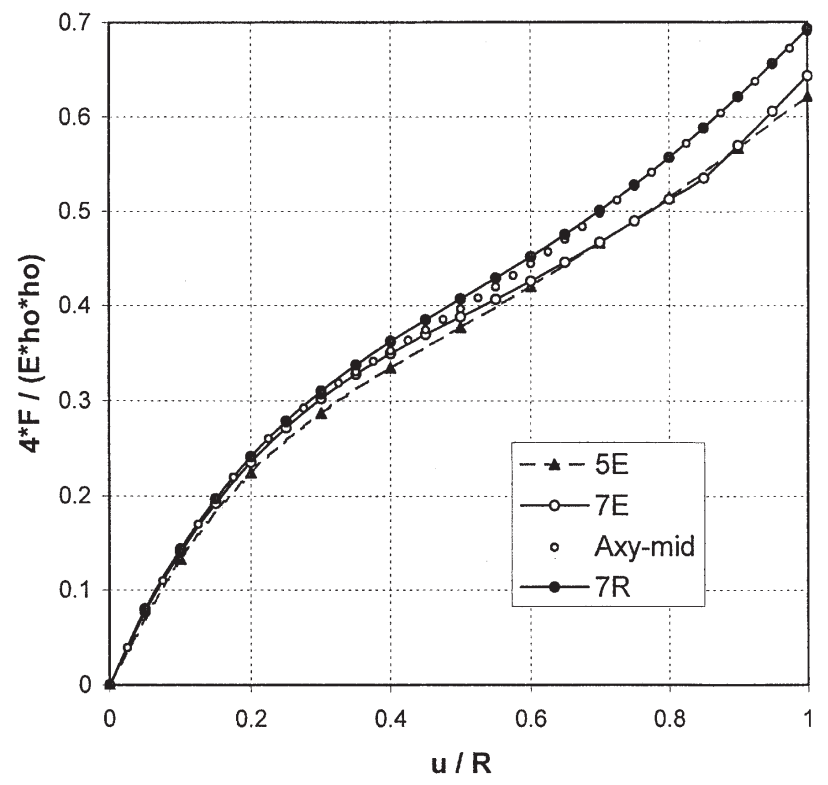

Fig. 12. Clamped sphere: normalized load versus normalized deflection curves for 7-parameter elements, $5 E$ element and axisymmetrical solution.

the NH material and with the load $F$ distributed at the middle surface over an interval of $0.01 R$. Note that the $7 R$ and the axisymmetrical solutions are very close to each other and that the $6 E A$ and the $6 E M$ solutions are on one side of the $5 E$ curve, while the $7 E$ and the $7 R$ solutions are on the opposite side of that curve. This can be due to very localized effect of thickness change which can be observed from Fig. 15, where practically all significant thickness stretch is restricted to one finite element.

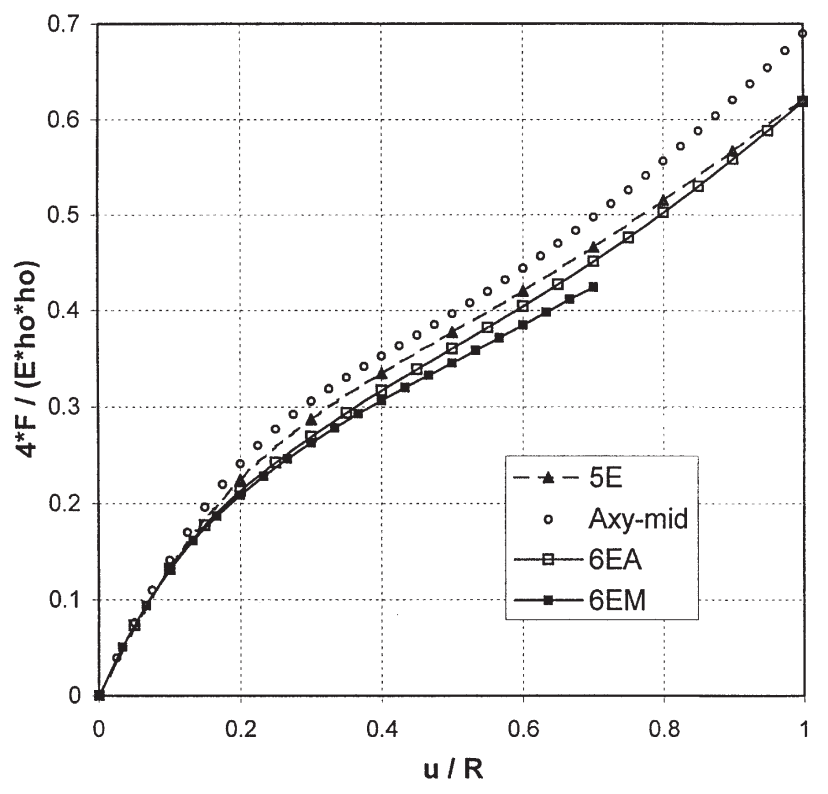

Fig. 13. Clamped sphere: normalized load versus normalized deflection curves for 6-parameter elements, $5 E$ element and axisymmetrical solution.

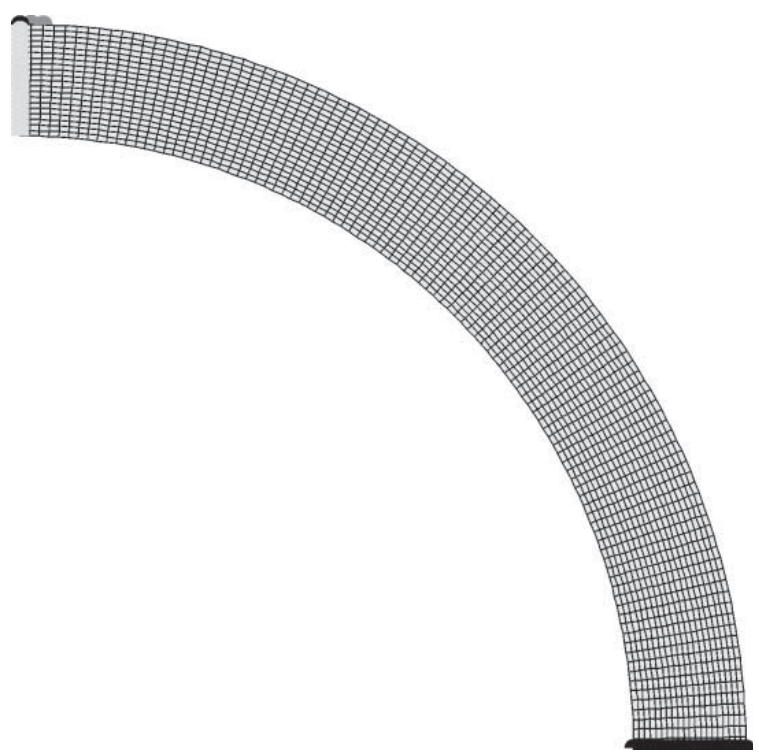

Fig. 14. Clamped sphere: a mesh of axisymmetrical elements; load is applied at the top surface.

In Table 8 the thickness change at the loading point is given for the last correctly converged solution. The $7 E$ and the $6 A E$ elements predict that the thickness of the final configuration would be $19 \%$ and $11 \%$, respectively, of the initial one. In Fig. 16 we present a distribution of $q$ over a sphere, which is very symmetric. Finally, Fig. 17 compares results obtained by the axisymmetrical element ("Axy-mid" refers to a case when force is applied on the middle surface, and "Axy-top" refers to a case when force is applied on the top surface) with the ex- 
Min: -0.0595818 Maxı 0808241

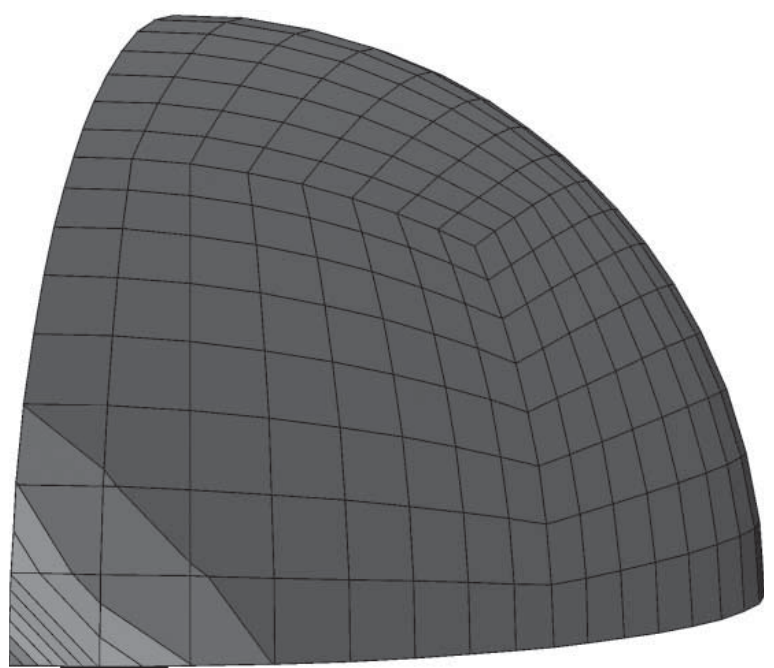

Fig. 15. Clamped sphere: distribution of thickness change $\tilde{\lambda}$ for $7 R$ element at $u / R=1$. Significant thickness change is very localized.

Table 8

Clamped sphere; values of $\tilde{\lambda}$ and $q$ at the loading point for the last correctly converged configuration

\begin{tabular}{llll}
\hline Element & $u / R$ & $\tilde{\lambda}$ & $q$ \\
\hline SVK material & & & \\
$5 E$ & 1.0 & - & - \\
$7 E$ & 0.8 & 0.64 & -0.021 \\
$7 R$ & 1.0 & 0.81 & 0.002 \\
$6 E A$ & 1.0 & 0.89 & - \\
$6 E M$ & 0.7 & 0.78 & - \\
\hline
\end{tabular}

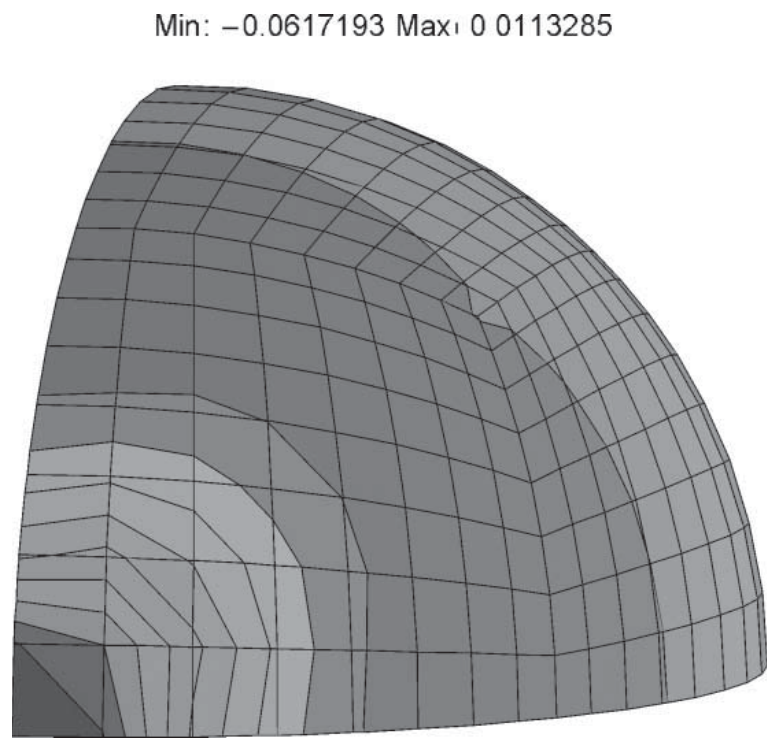

Fig. 16. Clamped sphere: distribution of $q$ variable over the sphere for $7 R$ element at $u / R=0.3$.

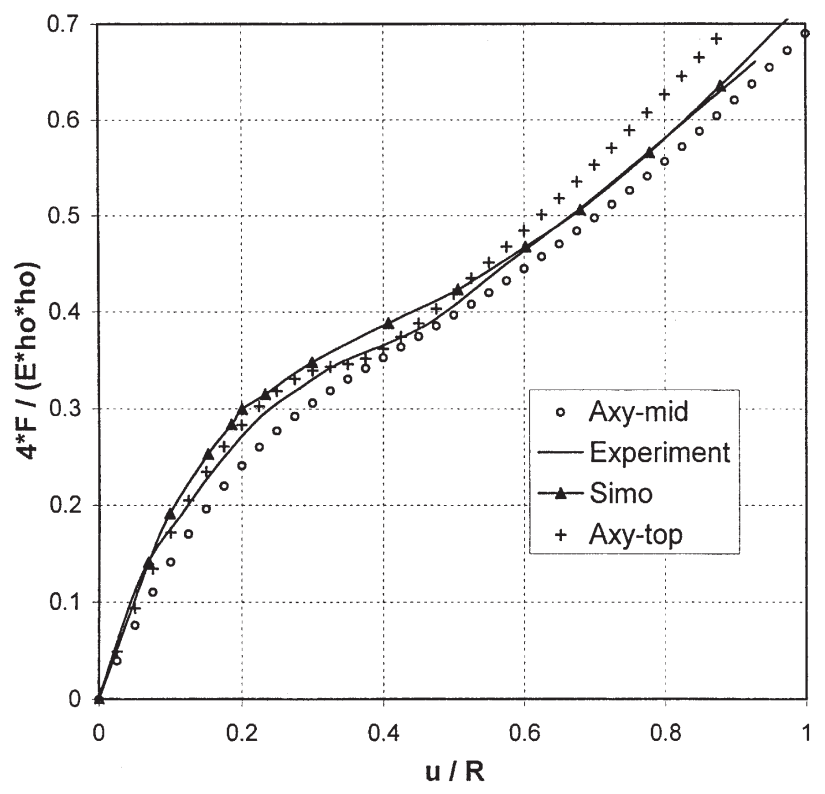

Fig. 17. Clamped sphere: comparison of axisymmetrical results with those obtained by the experiment and by Simo et al. [30].

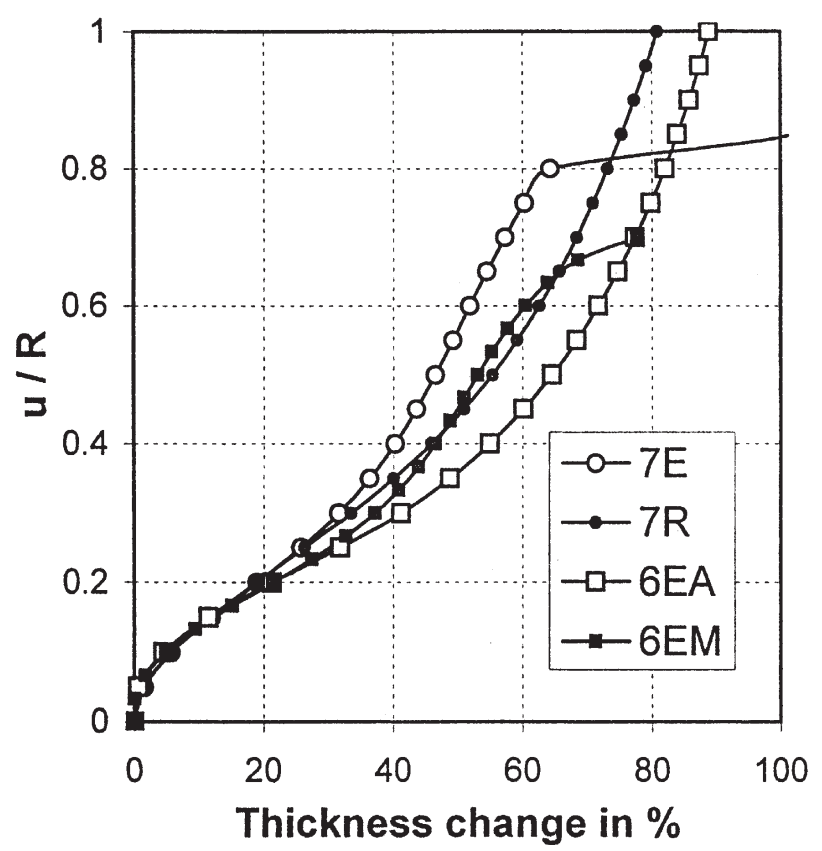

Fig. 18. Clamped sphere: thickness change $\tilde{\lambda}$ with respect to normalized displacement at the node under the force.

perimental data taken from the work of Simo et al. [30] and solution obtained by the same authors. They used 5parameter element and eliminated the transverse shear deformations by multiplying the shear correction factor by 100 . Figs. 18 and 19 show the evolution of thickness change and $q$ variable under the concentrated load. Solutions are quite different which can be again explained by very localized effect of the through-thethickness stretching. Figs. 20 and 21 show the final 


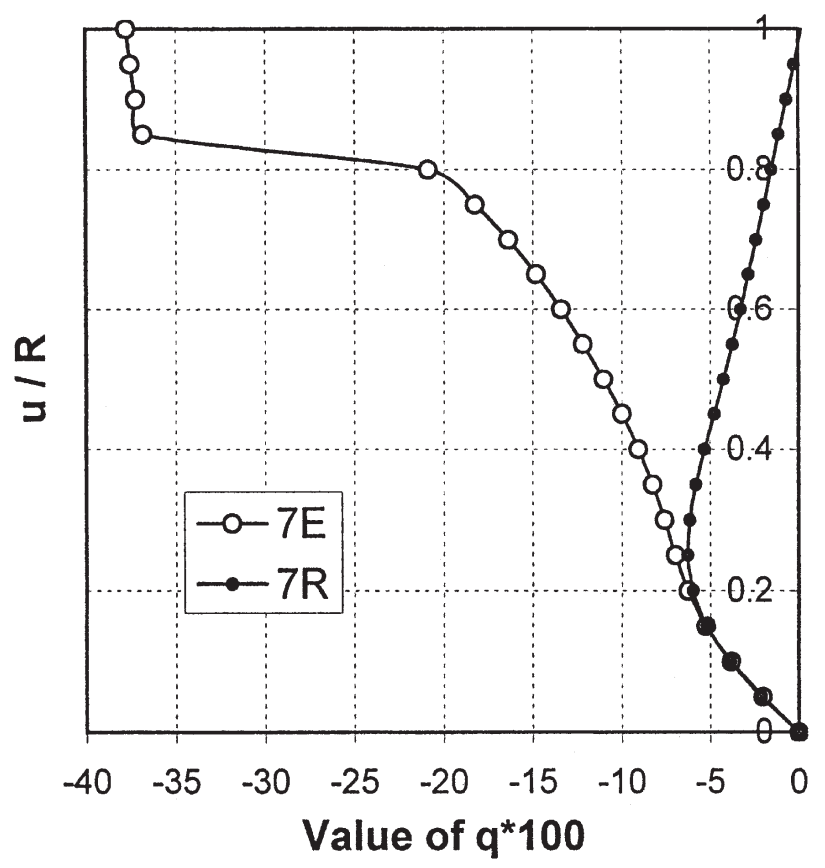

Fig. 19. Clamped sphere: $q$ variable with respect to normalized displacement at the node under the force.

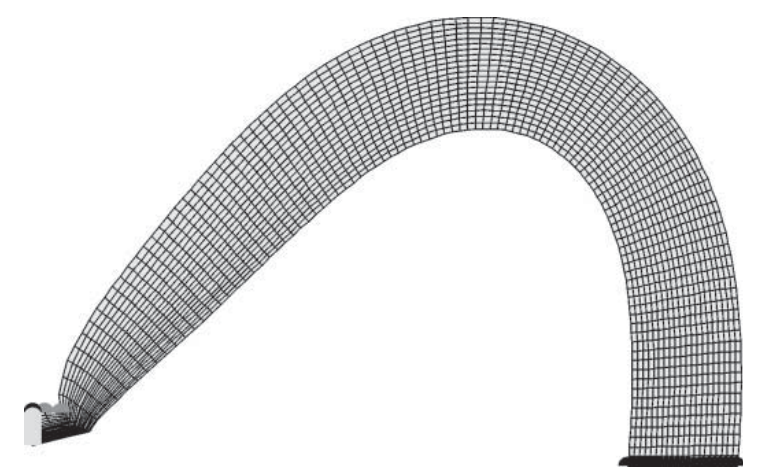

Fig. 20. Clamped sphere: axisymmetrical elements; top surface load; $u / R=1$.

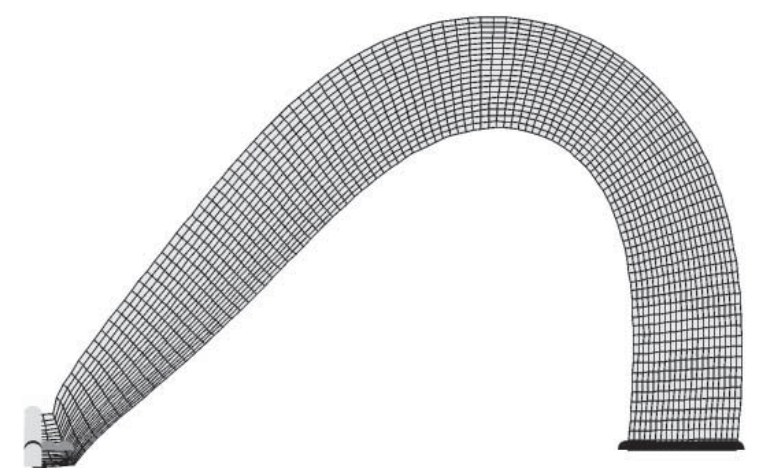

Fig. 21. Clamped sphere: axisymmetrical elements; middle surface load; $u / R=1$. configurations of axisymmetrical elements when the force is applied on the top surface and the middle surface, respectively. Significant difference in the deformation shape in the vicinity of the applied load can be observed.

\section{Conclusions}

Three nonlinear shell formulations accounting for through-the-thickness stretching were developed, one leading to a 7-parameter theory and the other two to theories with six parameters. All developed finite elements posses three displacement degrees of freedom and two rotations, and each one is capable of representing a linear variation of the through-the-thickness strain.

The 7-parameter shell theory is developed and tested in its full generality before reducing it to the usual format where only linear through-the-thickness variations of strain measures are kept. This kind of development is relatively easy to handle by symbolic manipulation. An incompatible mode method is used to reduce the 7parameter shell theory to the one with only six nodal parameters, which is easier to implement within the standard finite element computer program architecture. The main advantage of all presented shell models relates to a possibility to employ directly a $3 \mathrm{~d}$ form of constitutive equations with no presence of locking phenomena. Additional cures for locking employed by the derived elements include the assumed natural strain (ANS) method for the transverse shear locking, and the exact director vector interpolation for the curvature locking.

In the thin shell limit the elements with incompatible modes produce the same results as those obtained by the classical shell elements based on the Reissner-Mindlin kinematics (for the chosen numerical examples); i.e. the enhancement on through-the-thickness strain is not activated if not needed. The same is no longer true for the 7-parameter models which will not necessarily yield the same results as the 5-parameter model. For thick shells, the numerical results show that the influence of higherorder strains (which are usually neglected in shell theories) increases. In the computed examples there were no significant differences between two incompatible modes methods (one with an additive decomposition of strains and another with a multiplicative decomposition of strains) if thickness change was not extremely significant. The loading was applied at the shell middle surface in all numerical examples, however, the results indicate that the influence of the loading position to local results is quite important. 
Appendix A

Initialization and symbolic-numeric interface for tangent and residual user routine

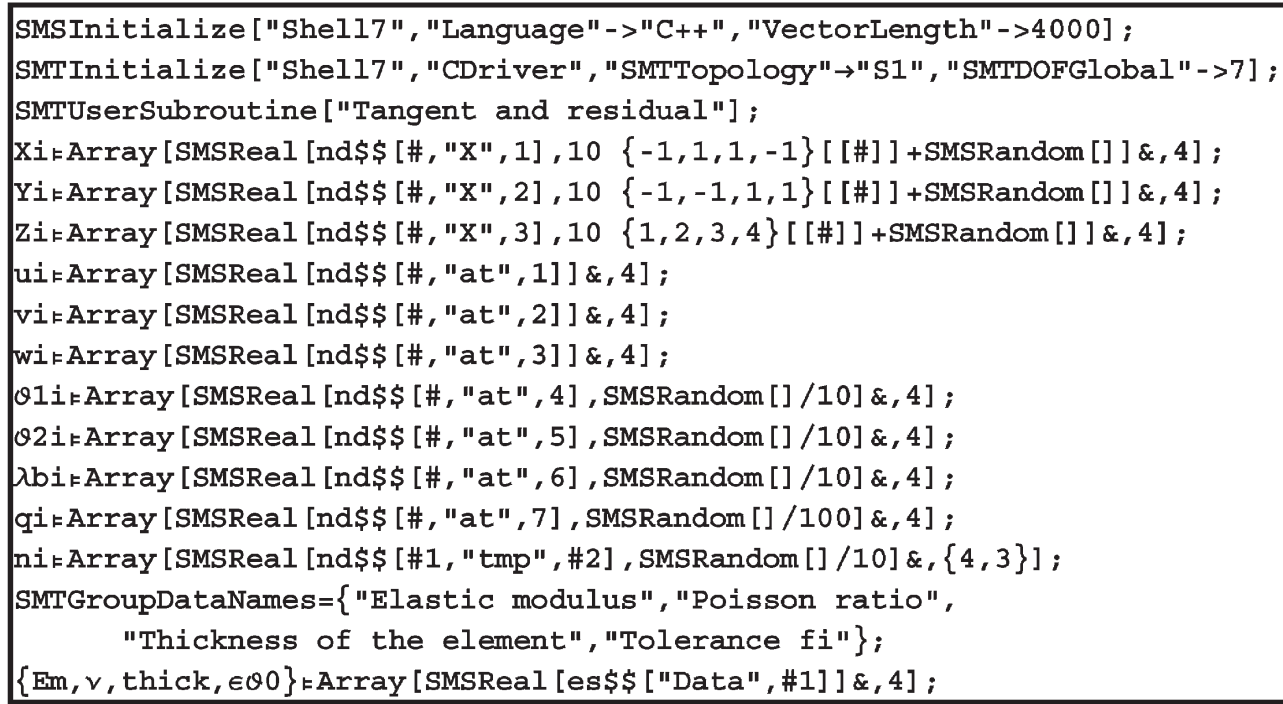

Nodal coordinate system and shell director

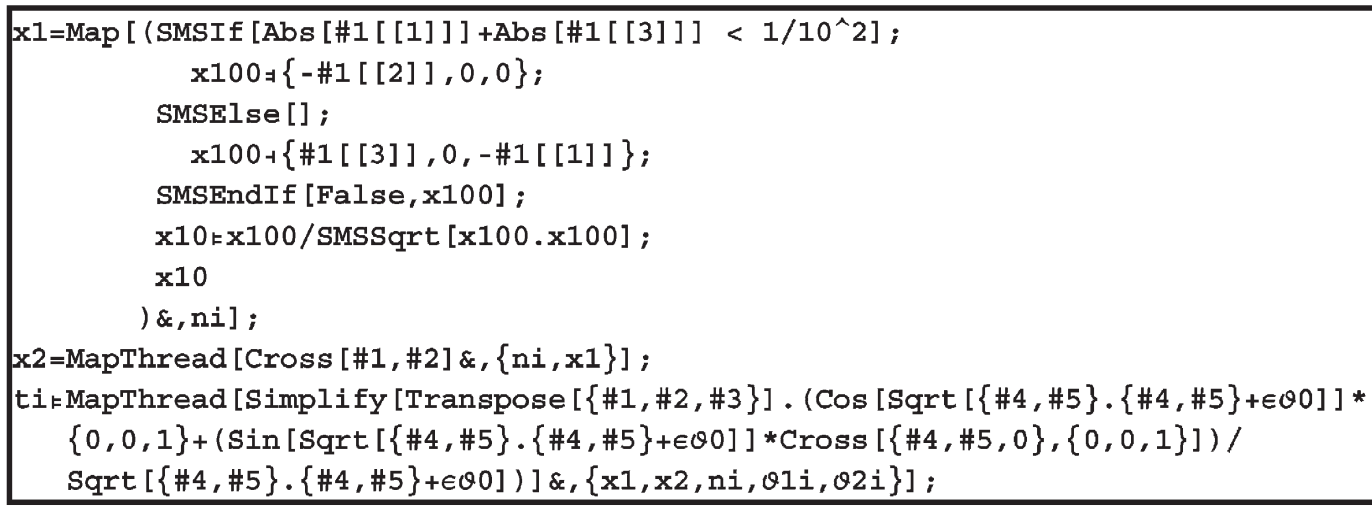

Integration loop and interpolations of nodal quantities

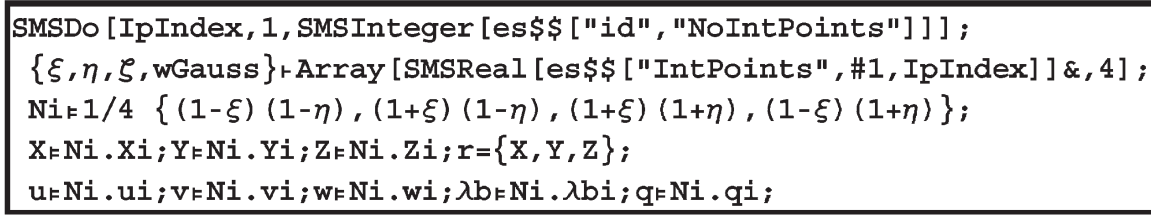

Coordinate system and shell director at integration point

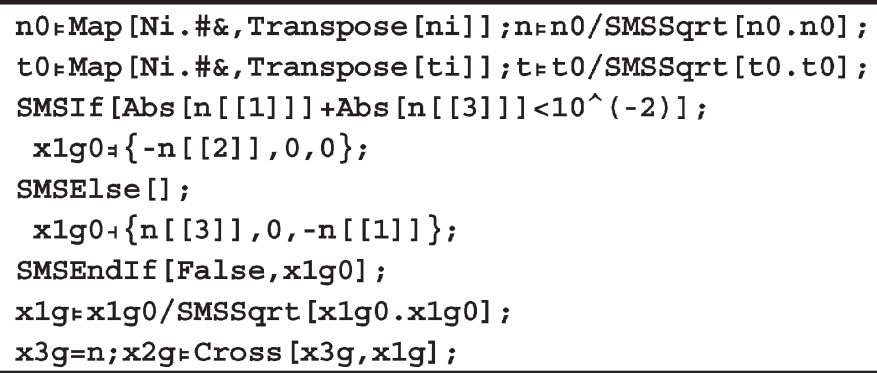




\section{Appendix B}

Shell kinematics

$\mathrm{x}=\mathrm{r}+\{\mathrm{u}, \mathrm{v}, \mathrm{w}\}+(\mathrm{thick} / 2) * \zeta *(1-\lambda \mathrm{b}) * \mathrm{t}+(\mathrm{thick} \wedge 2 / 4) * \zeta^{\wedge} 2 * \mathrm{q}^{*} \mathrm{t} ;$

Jmk SMSD $[r+($ thick $/ 2) * \zeta * n,\{\xi, \eta, \zeta\}] ; \operatorname{Jdk} \operatorname{Det}[\mathrm{Jm}]$;

$\mathbb{F} \vDash \operatorname{SMSD}[x,\{\xi, \eta, \zeta\}]$. Inverse $[\mathrm{Jm}] ; \mathbb{C} \in$ Transpose $[\mathbb{F}] . \mathbb{F} ;$

$\mathbb{C} I_{F}\{x 1 g, x 2 g, x 3 g\} . \mathbb{C}$.Transpose $[\{x 1 g, x 2 g, x 3 g\}]$;

$\mathbb{E} I_{k}(1 / 2) *(\mathbb{C} 1$-IdentityMatrix [3]);

ANS formulation

$r \xi \vDash \operatorname{SMSD}[r, \xi] ; r \eta$ F SMSD $[r, \eta]$;

$e \xi=(1 / 2) *(r \xi \cdot t *(1-\lambda b)+\operatorname{SMSD}[\{u, v, w\}, \xi] . t *(1-\lambda b)+(t h i c k / 2) *(\operatorname{SMSD}[q, \xi] *$

$\left.\left.(1-\lambda b)^{\wedge} 2-q^{*} \operatorname{SMSD}[\lambda b, \xi] *(1-\lambda b)\right)-r \xi \cdot n\right)$;

e $\eta=(1 / 2) *(r \eta \cdot t *(1-\lambda b)+\operatorname{SMSD}[\{u, v, w\}, \eta] . t *(1-\lambda b)+(\operatorname{thick} / 2) *(\operatorname{SMSD}[q, \eta] *$

$\left.\left.(1-\lambda b)^{\wedge} 2-q * \operatorname{SMSD}[\lambda b, \eta] *(1-\lambda b)\right)-r \eta \cdot n\right)$;

$\{\mathbb{E A}, \mathbb{E B}, \mathbb{E C}, \mathbb{E D}\}=$ (SMSSmartRestore $[\# 1[[3]], \xi|\eta| \zeta,\{\xi->\# 1[[1]], \eta->\# 1[[2]], \zeta->0\}] \&$ )/@ $\{\{0,-1, e \xi\},\{1,0, e \eta\},\{0,1, e \xi\},\{-1,0, e \eta\}\}$

e $\xi i=(1 / 2) *(1-\eta) * \mathbb{E} A+(1 / 2) *(1+\eta) * \mathbb{E} C ;$ e $\eta i_{k}(1 / 2) *(1-\xi) * \mathbb{E} D+(1 / 2) *(1+\xi) * \mathbb{E} B$;

ek Inverse $[\{\{r \xi \cdot x 1 g, r \xi \cdot x 2 g\},\{r \eta \cdot x 1 g, r \eta \cdot x 2 g\}\}] \cdot\{e \xi i, e \eta i\}$;

$\mathbb{E} 1[[1,3]]=\mathbb{E} 1[[3,1]]=e[[1]] ; \mathbb{E} 1[[2,3]]=\mathbb{E} 1[[3,2]]=e[[2]] ;$

Strain potentital energy for Neo-Hookean material

$\mathbb{C}=2 * \mathbb{E} 1+$ IdentityMatrix [3] ; $\mathbb{J}$ : Sqrt [Det [C] ] ;

$\{\lambda, \mu\}$ \& SMTHookeToLame $[\mathrm{Em}, v]$;

$\Pi_{F}(\lambda / 2) *(\mathbb{U}-1)^{\wedge} 2+\mu *((\operatorname{Tr}[\mathbb{C}]-3) / 2-\log [\mathbb{U}])$;

Formulation of residual vector and tangent matrix

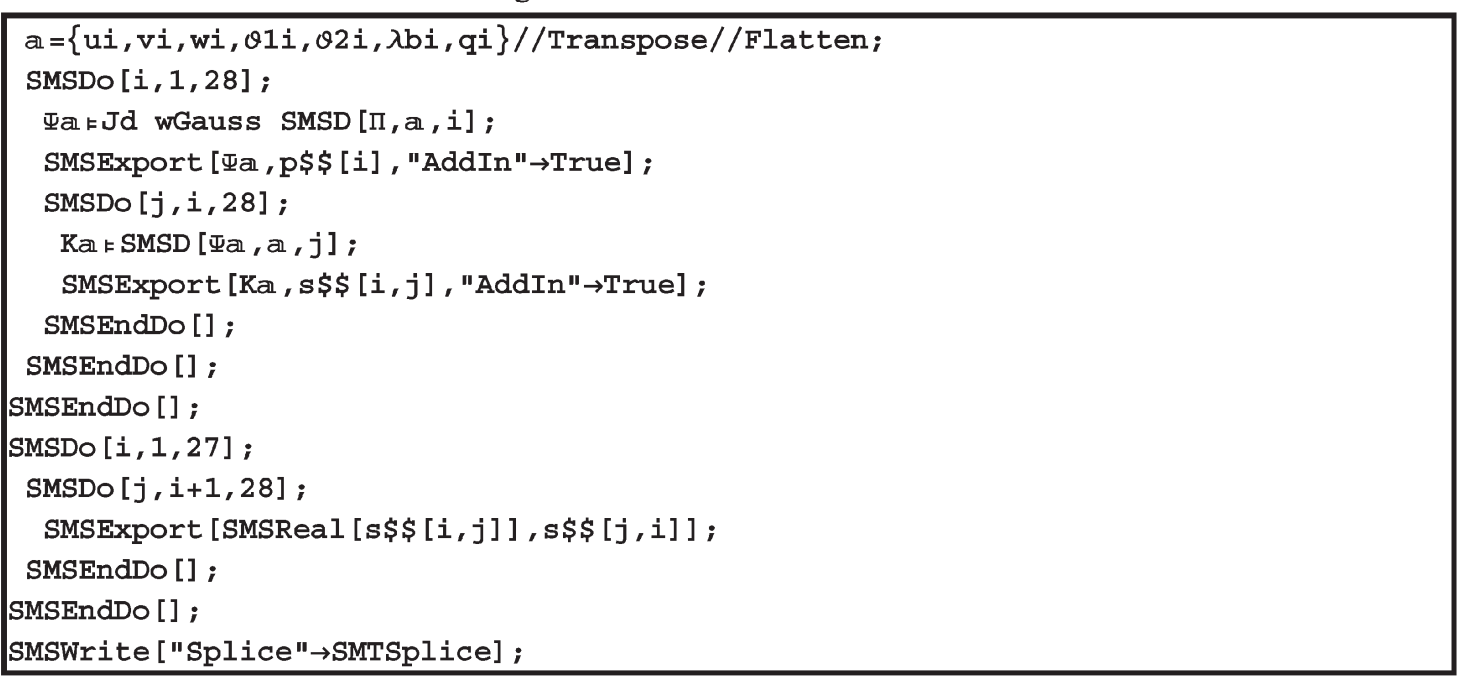

\section{References}

[1] El-Abbasi N, Meguid SA. A new shell element accounting for through-thickness deformation. Comput Meth Appl Mech Eng 2000;189:841-62.

[2] Başar Y, Ding Y. Shear deformation models for largestrain shell analysis. Int J Solids Struct 1997;34:1687-708.

[3] Başar Y, Itskov M, Eckstein A. Composite laminates: nonlinear interlaminar stress analysis by multi-layer shell elements. Comput Meth Appl Mech Eng 2000;185:367-97.
[4] Bathe KJ, Dvorkin E. A four-node plate bending element based on Mindlin-Reissner plate theory and a mixed interpolation. Int J Numer Meth Eng 1985;21:367-83.

[5] Betsch P, Stein E. An assumed strain approach avoiding artificial thickness straining for a non-linear 4-node shell element. Commun Numer Meth Eng 1995;11:899-909.

[6] Betsch P, Gruttmann F, Stein E. A 4-node finite shell element for the implementation of general hyperelastic 3Delasticity at finite strains. Comput Meth Appl Mech Eng 1996;130:57-79. 
[7] Bischoff M, Ramm E. Shear deformable shell elements for large strains and rotations. Int J Numer Meth Eng 1997;40: 4427-49.

[8] Bischoff M, Ramm E. On the physical significance of higher order kinematic and static variables in a threedimensional shell formulation. Int J Solids Struct 2000;37: 6933-60.

[9] Brank B, Perić D, Damjanić FB. On implementation of a nonlinear four node shell finite element for thin multilayered elastic shells. Comput Mech 1995;16:341-59.

[10] Brank B, Perić D, Damjanić FB. On large deformations of thin elasto-plastic shells: Implementation of a finite rotation model for quadrilateral shell element. Int $\mathbf{J}$ Numer Meth Eng 1997;40:689-726.

[11] Brank B, Ibrahimbegović A. On the relation between different parametrizations of finite rotations for shells. Eng Comput 2001;18:950-73.

[12] Büchter N, Ramm E. Shell theory versus degeneration - a comparison in large rotation finite element analysis. Int $\mathbf{J}$ Numer Meth Eng 1992;34:39-59.

[13] Büchter N, Ramm E, Roehl D. Three-dimensional extension of nonlinear shell formulation based on the enhanced assumed strain concept. Int J Numer Meth Eng 1994;37: 2551-68.

[14] Eberlein R, Wriggers P, Taylor RL. A fully non-linear axisymmetrical quasi-Kirchhoff-type shell element for rubber-like materials. Int J Numer Meth Eng 1993;36: 4027-43.

[15] Hauptmann R, Doll S, Harnau M, Schweizerhof K. 'Solidshell' elements with linear and quadratic shape functions at large deformations with nearly incompressible materials. Comput Struct 2001;79:1671-85.

[16] Ibrahimbegović A, Wilson EI. A modified method of incompatible modes. Commun Numer Meth Eng 1991;8: 23-32.

[17] Ibrahimbegović A, Frey F. Geometrically non-linear method of incompatible modes in application to finite elasticity with independent rotations. Int $\mathrm{J}$ Numer Meth Eng 1993;36:4185-200.

[18] Ibrahimbegović A. On assumed shear strain in finite rotation shell analysis. Eng Comput 1995;12:425-38.

[19] Ibrahimbegović A. Stress resultant geometrically exact shell theory for finite rotations and its finite element implementation. Appl Mech Rev 1997;50:199-226.

[20] Ibrahimbegović A, Brank B, Courtois P. Stress resultant geometrically exact form of classical shell model and vector-like parametrization of constrained finite rotations. Int J Numer Meth Eng 2001;52:1235-52.

[21] Klinkel S, Gruttmann F, Wagner W. A continuum based three-dimensional shell element for laminated structures. Comput Struct 1999;71:43-62.

[22] Korelc J. Automatic generation of finite element code by simultaneous optimization of expressions. Theor Comput Sci 1997;187:231-48.

[23] Korelc J. Automatic generation of numerical codes with introduction to AceGen 4.0 symbolic code generator, Faculty of Civil and Geodetic Engineering, University of Ljubljana, 2000. Available from: www.fgg.uni-lj.si/Symech/.

[24] Korelc J. Hybrid system for multi-language and multienvironment generation of numerical codes. In: Mourrain B, editor. Proceedings of the 2001 International Symposium on Symbolic and Algebraic Computation. The Association for Computing Machinery; 2001. p. 209-16.

[25] Krätzig WB. "Best" transverse shearing and stretching shell theory for nonlinear finite element simulations. Comput Meth Appl Mech Eng 1993;103:135-60.

[26] Naghdi PM. The theory of shells and plates. In: Flugge S, editor. Encyclopedia of physics. New York: Springer; 1972.

[27] Parisch H. A continuum-based shell theory for non-linear applications. Int J Numer Meth Eng 1995;38:1855-83.

[28] Sansour C. A theory and finite element formulation of shells at finite deformations involving thickness change: circumventing the use of a rotation tensor. Arch Appl Mech 1995;65:194-216.

[29] Simo JC, Rifai MS, Fox DD. On a stress resultant geometrically exact shell model. Part IV: Variable thickness shells with through-the-thickness stretching. Comput Meth Appl Mech Eng 1990;81:91-126.

[30] Simo JC, Rifai MS, Fox DD. On a stress resultant geometrically exact shell model. Part III: Computational aspects of the nonlinear theory. Comput Meth Appl Mech Eng 1990;79:21-70.

[31] Simo JC, Fox DD, Rifai MS. A class of mixed assumed strain methods and the method of incompatible modes. Int J Numer Meth Eng 1990;29:1595-638.

[32] Taylor RL, Simo JC, Zienkiewicz OC, Chan AC. The patch test: a condition for assessing finite element convergence. Int J Numer Meth Eng 1986;22:39-62.

[33] Zienkiewicz OC, Taylor RL. In: The finite element method: basic formulation and linear problems, vol. 1. New York: McGraw-Hill; 1989. 